\title{
Initial boundary value problem for generalized Zakharov equations with nonlinear function terms
}

\author{
Xueqin Wang ${ }^{1}$, Yadong Shang ${ }^{2 *}$ and Chunlin Lei'
}

\section{"Correspondence:}

gzydshang@126.com

${ }^{2}$ School of Mathematics and

Information Science, Guangzhou

University, Guangzhou, China

Full list of author information is

available at the end of the article

\begin{abstract}
In this paper, we consider the initial boundary value problem for generalized Zakharov equations. Firstly, we prove the existence and uniqueness of the global smooth solution to the problem by a priori integral estimates, the Galerkin method, and compactness theory. Furthermore, we discuss the approximation limit of the global solution when the coefficient of the strong nonlinear term tends to zero.

Keywords: Generalized Zakharov equations; Initial boundary value; Existence and uniqueness; Global smooth solution; Approximation of solution
\end{abstract}

\section{Introduction}

The Zakharov system, derived by Zakharov in 1972 [1], describes the interaction between Langmuir (dispersive) and ion acoustic (approximately nondispersive) waves in an unmagnetized plasma. The usual Zakharov system defined in the space $\mathbb{R}^{d+1}$ is given by

$$
\begin{aligned}
& i \varepsilon_{t}+\Delta \varepsilon=n \varepsilon, \\
& n_{t t}-\Delta n=\Delta|\varepsilon|^{2},
\end{aligned}
$$

where the wave fields $\varepsilon(x, t)$ and $n(x, t)$ are complex and real, respectively. It has become commonly accepted that the Zakharov system is a general model to govern interaction of dispersive and nondispersive waves.

The generalized Zakharov system has found a number of applications in various physical problems, such as interaction of intramolecular vibrations giving rise to Davydov solitons with acoustic disturbances [2], interaction of high- and low-frequency gravity disturbances in an atmosphere [3], and so on. In the past decades, the Zakharov system has been studied by many authors [4-13].

Gajewski and Zacharias [14] studied the following generalized Zakharov system and established the global existence for initial value problem:

$$
i \varepsilon_{t}+\varepsilon_{x x}+(\alpha-n) \varepsilon=0
$$

(c) The Author(s) 2020. This article is licensed under a Creative Commons Attribution 4.0 International License, which permits use, sharing, adaptation, distribution and reproduction in any medium or format, as long as you give appropriate credit to the original author(s) and the source, provide a link to the Creative Commons licence, and indicate if changes were made. The images or other third party material in this article are included in the article's Creative Commons licence, unless indicated otherwise in a credit line to the material. If material is not included in the article's Creative Commons licence and your intended use is not permitted by statutory regulation or exceeds the permitted use, you will need to obtain permission directly from the copyright holder. To view a copy of this licence, visit http://creativecommons.org/licenses/by/4.0/. 


$$
\begin{aligned}
& v_{t}+\left(\frac{1}{2} v^{2}-\beta v_{x}+n+|\varepsilon|^{2}\right)_{x}=0, \\
& n_{t}+v_{x}=0, \quad t>0,
\end{aligned}
$$

where the parameters $\beta>0$ and $\alpha$ are real numbers.

You and Ning [15] considered the existence and uniqueness of the global smooth solution for the initial value problem of the following generalized Zakharov equations in dimension two:

$$
\begin{aligned}
& i \varepsilon_{t}+\Delta \varepsilon-n \varepsilon=0, \\
& v_{t}+\sum_{i=1}^{2} \frac{\partial}{\partial x_{i}} \operatorname{grad} \varphi(v)-\Delta v+\nabla\left(n+|\varepsilon|^{2}\right)=0, \\
& n_{t}+\nabla \cdot v=0, \quad t>0,
\end{aligned}
$$

with initial data

$$
\left.\varepsilon\right|_{t=0}=\varepsilon_{0}(x),\left.\quad v\right|_{t=0}=v_{0}(x),\left.\quad n\right|_{t=0}=n_{0}(x),
$$

where $\varepsilon(x, t)=\left(\varepsilon_{1}(x, t), \varepsilon_{2}(x, t), \ldots, \varepsilon_{N}(x, t)\right)$ is an $N$-dimensional complex-valued unknown functional vector, $v(x, t)=\left(v_{1}(x, t), v_{2}(x, t)\right)$ is a two-dimensional real-valued unknown functional vector, $n(x, t)$ is a real-valued unknown function, $x \in \mathbb{R}^{2}$, and $\varphi(s)$ is a real function.

In the present paper, we study the following initial boundary value problem for generalized Zakharov equations:

$$
\begin{aligned}
& i \varepsilon_{t}+\varepsilon_{x x}+(\alpha-n) \varepsilon+\delta|\varepsilon|^{p} \varepsilon=0, \\
& v_{t}+\left[\varphi(v)-\beta v_{x}+n+|\varepsilon|^{2}\right]_{x}=0, \\
& n_{t}+v_{x}=0, \quad t>0, x \in[0, L],
\end{aligned}
$$

with initial data

$$
\left.\varepsilon\right|_{t=0}=\varepsilon_{0}(x),\left.\quad v\right|_{t=0}=v_{0}(x),\left.\quad n\right|_{t=0}=n_{0}(x), \quad x \in[0, L],
$$

and boundary conditions

$$
\varepsilon(0, t)=\varepsilon(L, t)=v(0, t)=v(L, t)=n(0, t)=n(L, t)=0,
$$

where the parameters $p>0, \beta>0, \alpha$, and $\delta$ are real numbers, and $\varphi(s)$ is a real function. Taking $\delta=0, \beta=0$, and $\varphi^{\prime}(s)=$ Constant in this system, it becomes the classical Zakharov equation system. From a physical point of view, this system has stronger nonlinear excitation and interaction. It also can be considered as a further generalization of the generalized Zakharov system discussed in [14]. From the perspective of both mathematical research and physical applications, the problem is of great significance.

For convenience of the following contexts, we set some notations. For $1 \leq p \leq \infty$, we denote by $L^{p}[0, L]$ the space of all $p$ th-power integrable functions in $[0, L]$ equipped with 
norm $\|\cdot\|_{L^{p}}$, and by $H^{s, p}$ the Sobolev space with norm $\|\cdot\|_{H^{s, p}}$. For $p=2$, we write $H^{s}$ instead of $H^{s, 2} . C^{k}(R)$ is the space of $k$ times continuously differentiable functions on $R$. If $k=0$, then we write $C(R)$ instead of $C^{0}(R)$. Let $(f, g)=\int_{0}^{L} f(x) \overline{g(x)} d x$, where $\overline{g(x)}$ denotes the complex conjugate function of $g(x)$. The real and imaginary parts of a complex number $A$ are denoted, respectively, by $\operatorname{Re} A$ and $\operatorname{Im} A$. Throughout the paper, $C$ is a generic constant, which may have different meanings in different places.

This paper is organized as follows. In Sect. 2, we establish a priori estimations for problem (1.1)-(1.5). In Sect. 3, we study the existence and uniqueness of global generalized solutions for problem (1.1)-(1.5). In Sect. 4, we discuss the regularity of global generalized solution for problem (1.1)-(1.5). In Sect. 5, we give the approximation limit of the global solution when the coefficient of the strong nonlinear term tends to zero.

\section{A priori estimations for problem (1.1)-(1.5)}

Lemma 2.1 Let $\varepsilon_{0} \in L^{2}[0, L]$. Then for the solution of problem (1.1)-(1.5), we have

$$
\|\varepsilon\|_{L^{2}}^{2}=\left\|\varepsilon_{0}\right\|_{L^{2}}^{2}
$$

Proof Taking the inner product of (1.1) and $\varepsilon$, we have

$$
\left(i \varepsilon_{t}+\varepsilon_{x x}+(\alpha-n) \varepsilon+\delta|\varepsilon|^{p} \varepsilon, \varepsilon\right)=0 .
$$

Since $\operatorname{Im}\left(i \varepsilon_{t}, \varepsilon\right)=\frac{1}{2} \frac{d}{d t}\|\varepsilon\|_{L^{2}}^{2}, \operatorname{Im}\left(\varepsilon_{x x}+(\alpha-n) \varepsilon+\delta|\varepsilon|^{p} \varepsilon, \varepsilon\right)=0$, and hence from (2.1) we get

$$
\frac{d}{d t}\|\varepsilon\|_{L^{2}}^{2}=0
$$

that is,

$$
\|\varepsilon\|_{L^{2}}^{2}=\left\|\varepsilon_{0}\right\|_{L^{2}}^{2}
$$

Lemma 2.2 Suppose that (1) $\varepsilon_{0} \in H^{1}[0, L], v_{0} \in L^{2}[0, L], n_{0} \in L^{2}[0, L]$, and (2) $\varphi(v) \in C(R)$.

Then for the solution of problem (1.1)-(1.5), we have

$$
\begin{aligned}
& \left\|\varepsilon_{x}\right\|_{L^{2}}^{2}+\int_{0}^{L} n|\varepsilon|^{2} d x-\frac{2 \delta}{p+2} \int_{0}^{L}|\varepsilon|^{p+2} d x+\frac{1}{2}\|v\|_{L^{2}}^{2}+\frac{1}{2}\|n\|_{L^{2}}^{2} \\
& \quad+\beta \int_{0}^{t}\left\|v_{x}(x, \tau)\right\|_{L^{2}}^{2} d \tau=M_{1} .
\end{aligned}
$$

Proof Taking the inner product of (1.1) and $-\varepsilon_{t}$, we get that

$$
\left(i \varepsilon_{t}+\varepsilon_{x x}+(\alpha-n) \varepsilon+\delta|\varepsilon|^{p} \varepsilon,-\varepsilon_{t}\right)=0 .
$$

Since

$$
\operatorname{Re}\left(i \varepsilon_{t},-\varepsilon_{t}\right)=0, \operatorname{Re}\left(\varepsilon_{x x},-\varepsilon_{t}\right)=\frac{1}{2} \frac{d}{d t}\left\|\varepsilon_{x}\right\|_{L^{2}}^{2}
$$


we have

$$
\begin{aligned}
\operatorname{Re}\left((\alpha-n) \varepsilon,-\varepsilon_{t}\right)= & -\frac{\alpha}{2} \int_{0}^{L}\left(\varepsilon \bar{\varepsilon}_{t}+\bar{\varepsilon} \varepsilon_{t}\right) d x+\frac{1}{2} \frac{d}{d t} \int_{0}^{L} n|\varepsilon|^{2} d x \\
& -\frac{1}{2} \int_{0}^{L} n_{t}|\varepsilon|^{2} d x \\
= & -\frac{\alpha}{2} \frac{d}{d t}\|\varepsilon\|_{L^{2}}^{2}+\frac{1}{2} \frac{d}{d t} \int_{0}^{L} n|\varepsilon|^{2} d x-\frac{1}{2} \int_{0}^{L} n_{t}|\varepsilon|^{2} d x \\
= & \frac{1}{2} \frac{d}{d t} \int_{0}^{L} n|\varepsilon|^{2} d x-\frac{1}{2} \int_{0}^{L} n_{t}|\varepsilon|^{2} d x, \\
\operatorname{Re}\left(\delta|\varepsilon|^{p} \varepsilon,-\varepsilon_{t}\right)= & -\frac{\delta}{p+2} \frac{d}{d t} \int_{0}^{L}|\varepsilon|^{p+2} d x,
\end{aligned}
$$

and hence from (2.2) we get

$$
\frac{d}{d t}\left(\left\|\varepsilon_{x}\right\|_{L^{2}}^{2}+\int_{0}^{L} n|\varepsilon|^{2} d x-\frac{2 \delta}{p+2} \int_{0}^{L}|\varepsilon|^{p+2} d x\right)-\int_{0}^{L} n_{t}|\varepsilon|^{2} d x=0 .
$$

Taking the inner product of (1.2) and $v$, we have

$$
\left(v_{t}+\left[\varphi(v)-\beta v_{x}+n+|\varepsilon|^{2}\right]_{x}, v\right)=0 .
$$

Since

$$
\begin{aligned}
& \left(v_{t}, v\right)=\frac{1}{2} \frac{d}{d t}\|v\|_{L^{2}}^{2}, \quad\left(-\beta v_{x x}, v\right)=\beta\left\|v_{x}\right\|_{L^{2}}^{2}, \\
& \left([\varphi(v)]_{x^{\prime}}, v\right)=\int_{0}^{L}[\varphi(v)]_{x} v d x=-\frac{1}{2} \int_{0}^{L} \varphi(v) v_{x} d x=-\left.\frac{1}{2} \Phi(v(x, t))\right|_{0} ^{L}=0
\end{aligned}
$$

where

$$
\begin{aligned}
& \Phi(x)=\int_{0}^{x} \varphi(s) d s \\
& \left(n_{x}, v\right)=-\int_{0}^{L} n v_{x} d x=\int_{0}^{L} n n_{t} d x=\frac{1}{2} \frac{d}{d t}\|n\|_{L^{2}}^{2}, \\
& \left(|\varepsilon|_{x}^{2}, v\right)=-\int_{0}^{L}|\varepsilon|^{2} v_{x} d x=\int_{0}^{L}|\varepsilon|^{2} n_{t} d x
\end{aligned}
$$

and hence from (2.4) we get

$$
\frac{d}{d t}\left(\frac{1}{2}\|v\|_{L^{2}}^{2}+\frac{1}{2}\|n\|_{L^{2}}^{2}\right)+\int_{0}^{L}|\varepsilon|^{2} n_{t} d x+\beta\left\|v_{x}\right\|_{L^{2}}^{2}=0 .
$$

By (2.3) and (2.5) we get

$$
\begin{aligned}
& \frac{d}{d t}\left(\left\|\varepsilon_{x}\right\|_{L^{2}}^{2}+\int_{0}^{L} n|\varepsilon|^{2} d x-\frac{2 \delta}{p+2} \int_{0}^{L}|\varepsilon|^{p+2} d x+\frac{1}{2}\|v\|_{L^{2}}^{2}\right) \\
& \quad+\frac{d}{d t}\left(\frac{1}{2}\|n\|_{L^{2}}^{2}\right)+\beta\left\|v_{x}\right\|_{L^{2}}^{2}=0 .
\end{aligned}
$$


Thus

$$
\begin{aligned}
\left\|\varepsilon_{x}\right\|_{L^{2}}^{2} & +\int_{0}^{L} n|\varepsilon|^{2} d x-\frac{2 \delta}{p+2} \int_{0}^{L}|\varepsilon|^{p+2} d x \\
& +\frac{1}{2}\|v\|_{L^{2}}^{2}+\frac{1}{2}\|n\|_{L^{2}}^{2}+\beta \int_{0}^{t}\left\|v_{x}(x, \tau)\right\|_{L^{2}}^{2} d \tau \\
= & \left\|\varepsilon_{0 x}\right\|_{L^{2}}^{2}+\int_{0}^{L} n_{0}\left|\varepsilon_{0}\right|^{2} d x-\frac{2 \delta}{p+2} \int_{0}^{L}\left|\varepsilon_{0}\right|^{p+2} d x \\
& +\frac{1}{2}\left\|v_{0}\right\|_{L^{2}}^{2}+\frac{1}{2}\left\|n_{0}\right\|_{L^{2}}^{2} \\
= & M_{1} .
\end{aligned}
$$

Lemma 2.3 (Sobolev estimates)

(1) Assuming that $u \in L^{q}\left(R^{n}\right), D^{m} u \in L^{r}\left(R^{n}\right), 1 \leq q, r \leq \infty, 0 \leq j<m$, we have the estimates

$$
\left\|D^{j} u\right\|_{L^{p}} \leq C\left\|D^{m} u\right\|_{L^{r}}^{\theta}\|u\|_{L^{q}}^{1-\theta}
$$

where

$$
\frac{1}{p}=\frac{j}{n}+\theta\left(\frac{1}{r}-\frac{m}{n}\right)+(1-\theta) \frac{1}{q}, \quad \frac{j}{m} \leq \theta<1,
$$

and $C$ is a positive constant depending only on $n, m, j, q, r$, and $\theta$.

(2) For $\gamma>0$ and $s \in Z^{+}$, we can get a constant $C$ (it only depends on $\gamma$ and s) such that

$$
\begin{aligned}
& \left\|\frac{\partial^{k} u}{\partial x^{k}}\right\|_{L^{\infty}} \leq C\|u\|_{L^{2}}+\gamma\left\|\frac{\partial^{s} u}{\partial x^{s}}\right\|_{L^{2}}, \quad k<s, \\
& \left\|\frac{\partial^{k} u}{\partial x^{k}}\right\|_{L^{2}} \leq C\|u\|_{L^{2}}+\gamma\left\|\frac{\partial^{s} u}{\partial x^{s}}\right\|_{L^{2}}, \quad k \leq s .
\end{aligned}
$$

Lemma 2.4 Suppose that the conditions of Lemma 2.2 are satisfied and $0<p<4$. Then for the solution of problem (1.1)-(1.5), we have

$$
\sup _{t \in[0, T]}\left(\|\varepsilon\|_{H^{1}}+\|v\|_{L^{2}}+\|n\|_{L^{2}}\right)+\beta \int_{0}^{T}\left\|v_{x}(x, t)\right\|_{L^{2}}^{2} d t \leq C .
$$

Proof From Lemmas 2.1-2.3 and Young's inequality we get

$$
\begin{aligned}
& \left\|\varepsilon_{x}\right\|_{L^{2}}^{2}+\frac{1}{2}\|v\|_{L^{2}}^{2}+\frac{1}{2}\|n\|_{L^{2}}^{2}+\beta \int_{0}^{t}\left\|v_{x}(x, \tau)\right\|_{L^{2}}^{2} d \tau \\
& \leq\left|M_{1}\right|+\left.\left.\left|\int_{0}^{L} n\right| \varepsilon\right|^{2} d x\left|+\frac{2|\delta|}{p+2} \int_{0}^{L}\right| \varepsilon\right|^{p+2} d x \\
& \quad \leq\left|M_{1}\right|+\|n\|_{L^{2}}\|\varepsilon\|_{L^{4}}^{2}+\frac{2|\delta|}{p+2}\|\varepsilon\|_{L^{p+2}}^{p+2} \\
& \quad \leq\left|M_{1}\right|+\|n\|_{L^{2}}\left(C\|\varepsilon\|_{L^{2}}^{\frac{3}{2}}\left\|\varepsilon_{x}\right\|_{L^{2}}^{\frac{1}{2}}\right)+\frac{2|\delta|}{p+2}\left(C\left\|\varepsilon_{x}\right\|_{L^{2}}^{\frac{p}{2}}\|\varepsilon\|_{L^{2}}^{\frac{p+4}{2(p+2)}}\right)
\end{aligned}
$$




$$
\begin{aligned}
& \leq\left|M_{1}\right|+\|n\|_{L^{2}}\left(C\|\varepsilon\|_{L^{2}}^{\frac{3}{2}}\left\|\varepsilon_{x}\right\|_{L^{2}}^{\frac{1}{2}}\right)+C\left\|\varepsilon_{x}\right\|_{L^{2}}^{\frac{p}{2}} \\
& \leq\left|M_{1}\right|+\frac{1}{4}\|n\|_{L^{2}}^{2}+C\left\|\varepsilon_{x}\right\|_{L^{2}}+\frac{1}{2}\left\|\varepsilon_{x}\right\|_{L^{2}}^{2}+C \\
& \leq\left|M_{1}\right|+\frac{1}{4}\|n\|_{L^{2}}^{2}+\frac{3}{4}\left\|\varepsilon_{x}\right\|_{L^{2}}^{2}+C \\
& \leq \frac{1}{4}\|n\|_{L^{2}}^{2}+\frac{3}{4}\left\|\varepsilon_{x}\right\|_{L^{2}}^{2}+C,
\end{aligned}
$$

and hence

$$
\left\|\varepsilon_{x}\right\|_{L^{2}}^{2}+\|v\|_{L^{2}}^{2}+\|n\|_{L^{2}}^{2}+\beta \int_{0}^{t}\left\|v_{x}(x, \tau)\right\|_{L^{2}}^{2} d \tau \leq C .
$$

By (2.6) it follows that

$$
\sup _{t \in[0, T]}\left(\|\varepsilon\|_{H^{1}}+\|v\|_{L^{2}}+\|n\|_{L^{2}}\right)+\beta \int_{0}^{T}\left\|v_{x}(x, t)\right\|_{L^{2}}^{2} d t \leq C
$$

Corollary 2.1 Suppose that the conditions of Lemma 2.4 are satisfied. Then we have

$$
\sup _{t \in[0, T]}\|\varepsilon\|_{L^{\infty}} \leq C
$$

Proof By Lemmas 2.3 and 2.4, the result of Corollary 2.1 is obvious.

Lemma 2.5 Suppose that the conditions of Lemma 2.4 are satisfied, and assume that (1) $\varepsilon_{0} \in H^{2}[0, L], v_{0} \in H^{1}[0, L], n_{0} \in H^{1}[0, L]$, and (2) $\varphi(v) \in C^{1}(R),\left|\varphi^{\prime}(v)\right| \leq C\left(|v|^{q}+1\right)$, $0 \leq q \leq 2$. Then for the solution of problem (1.1)-(1.5), we have

$$
\sup _{t \in[0, T]}\left(\|\varepsilon\|_{H^{2}}+\|v\|_{H^{1}}+\|n\|_{H^{1}}+\left\|\varepsilon_{t}\right\|_{L^{2}}+\left\|n_{t}\right\|_{L^{2}}\right)+\beta \int_{0}^{T}\left\|v_{x x}(x, t)\right\|_{L^{2}}^{2} d t \leq C .
$$

Proof Differentiating (1.1) with respect to $t$, we get

$$
i \varepsilon_{t t}+\varepsilon_{x x t}+\alpha \varepsilon_{t}-n_{t} \varepsilon-n \varepsilon_{t}+\left(\delta|\varepsilon|^{p} \varepsilon\right)_{t}=0 .
$$

Taking the inner product of (2.7) and $\varepsilon_{t}$, it follows that

$$
\left(i \varepsilon_{t t}+\varepsilon_{x x t}+\alpha \varepsilon_{t}-n_{t} \varepsilon-n \varepsilon_{t}+\left(\delta|\varepsilon|^{p} \varepsilon\right)_{t}, \varepsilon_{t}\right)=0 .
$$

Since

$$
\begin{aligned}
& \operatorname{Im}\left(i \varepsilon_{t t}, \varepsilon_{t}\right)=\frac{1}{2} \frac{d}{d t}\left\|\varepsilon_{t}\right\|_{L^{2}}^{2}, \quad \operatorname{Im}\left(\varepsilon_{x x t}+\alpha \varepsilon_{t}-n \varepsilon_{t}, \varepsilon_{t}\right)=0, \\
& \begin{aligned}
\operatorname{Im}\left(\left(\delta|\varepsilon|^{p} \varepsilon\right)_{t}, \varepsilon_{t}\right) & =\operatorname{Im} \int_{0}^{L}\left[\left(1+\frac{p}{2}\right) \delta|\varepsilon|^{p} \varepsilon_{t}+\frac{p \delta}{2}|\varepsilon|^{p-2} \varepsilon^{2} \bar{\varepsilon}_{t}\right] \bar{\varepsilon}_{t} d x \\
& =\frac{p \delta}{2} \operatorname{Im} \int_{0}^{L}|\varepsilon|^{p-2} \varepsilon^{2} \bar{\varepsilon}_{t}^{2} d x,
\end{aligned}
\end{aligned}
$$


and hence from (2.8), (1.3), and Corollary 2.1 we get

$$
\begin{aligned}
\frac{1}{2} \frac{d}{d t}\left\|\varepsilon_{t}\right\|_{L^{2}}^{2} & =\operatorname{Im}\left(n_{t} \varepsilon, \varepsilon_{t}\right)-\frac{p \delta}{2} \operatorname{Im} \int_{0}^{L}|\varepsilon|^{p-2} \varepsilon^{2} \bar{\varepsilon}_{t}^{2} d x \\
& \leq\|\varepsilon\|_{L^{\infty}}\left\|n_{t}\right\|_{L^{2}}\left\|\varepsilon_{t}\right\|_{L^{2}}+\frac{p|\delta|}{2}\|\varepsilon\|_{L^{\infty}}^{p}\left\|\varepsilon_{t}\right\|_{L^{2}}^{2} \\
& \leq C\left(\left\|n_{t}\right\|_{L^{2}}^{2}+\left\|\varepsilon_{t}\right\|_{L^{2}}^{2}\right) \\
& \leq C\left(\left\|v_{x}\right\|_{L^{2}}^{2}+\left\|\varepsilon_{t}\right\|_{L^{2}}^{2}\right)
\end{aligned}
$$

Taking the inner product of (1.2) and $-v_{x x}$, it follows that

$$
\left(v_{t}+\left[\varphi(v)-\beta v_{x}+n+|\varepsilon|^{2}\right]_{x},-v_{x x}\right)=0 .
$$

Since

$$
\begin{aligned}
& \left(v_{t},-v_{x x}\right)=\frac{1}{2} \frac{d}{d t}\left\|v_{x}\right\|_{L^{2}}^{2}, \quad\left(-\beta v_{x x},-v_{x x}\right)=\beta\left\|v_{x x}\right\|_{L^{2}}^{2}, \\
& \left|\left([\varphi(v)]_{x},-v_{x x}\right)\right|=\left|\int_{0}^{L} \varphi^{\prime}(v) v_{x} v_{x x} d x\right| \\
& \leq C \int_{0}^{L}\left(|v|^{q}+1\right)\left|v_{x}\right|\left|v_{x x}\right| d x \\
& \leq\left(\|v\|_{L^{4 q}}^{q}\left\|v_{x}\right\|_{L^{4}}+\left\|v_{x}\right\|_{L^{2}}\right)\left\|v_{x x}\right\|_{L^{2}} \\
& \leq C\left[\left(\|v\|_{L^{2}}^{\frac{3 q}{4}+\frac{1}{8}}\left\|v_{x x}\right\|_{L^{2}}^{\frac{q}{4}-\frac{1}{8}}\right)\left(\|v\|_{L^{2}}^{\frac{3}{8}}\left\|v_{x x}\right\|_{L^{2}}^{\frac{5}{8}}\right)\right]\left\|v_{x x}\right\|_{L^{2}} \\
& +C\left\|v_{x}\right\|_{L^{2}}\left\|v_{x x}\right\|_{L^{2}} \\
& \leq C\left\|v_{x x}\right\|_{L^{2}}^{\frac{q}{4}+\frac{3}{2}}+C\left\|v_{x}\right\|_{L^{2}}\left\|v_{x x}\right\|_{L^{2}} \\
& \leq \frac{\beta}{4}\left\|v_{x x}\right\|_{L^{2}}^{2}+C\left(\left\|v_{x}\right\|_{L^{2}}^{2}+1\right), \\
& \left(n_{x},-v_{x x}\right)=-\int_{0}^{L} n_{x} v_{x x} d x=\int_{0}^{L} n_{x} n_{x t} d x=\frac{1}{2} \frac{d}{d t}\left\|n_{x}\right\|_{L^{2}}^{2} \text {, } \\
& \left|\left(|\varepsilon|_{x}^{2},-v_{x x}\right)\right| \leq 2 \int_{0}^{L}|\varepsilon|\left|\varepsilon_{x}\right|\left|v_{x x}\right| d x \\
& \leq 2\|\varepsilon\|_{L^{\infty}}\left\|\varepsilon_{x}\right\|_{L^{2}}\left\|v_{x x}\right\|_{L^{2}} \\
& \leq \frac{\beta}{4}\left\|v_{x x}\right\|_{L^{2}}^{2}+C
\end{aligned}
$$

and hence from (2.10) we get

$$
\frac{d}{d t}\left(\left\|v_{x}\right\|_{L^{2}}^{2}+\left\|n_{x}\right\|_{L^{2}}^{2}\right)+\beta\left\|v_{x x}\right\|_{L^{2}}^{2} \leq C\left(\left\|v_{x}\right\|_{L^{2}}^{2}+1\right) .
$$

By (2.9) and (2.11) we obtain

$$
\frac{d}{d t}\left(\left\|\varepsilon_{t}\right\|_{L^{2}}^{2}+\left\|v_{x}\right\|_{L^{2}}^{2}+\left\|n_{x}\right\|_{L^{2}}^{2}\right)+\beta\left\|v_{x x}\right\|_{L^{2}}^{2} \leq C\left(\left\|v_{x}\right\|_{L^{2}}^{2}+\left\|\varepsilon_{t}\right\|_{L^{2}}^{2}+\left\|n_{x}\right\|_{L^{2}}^{2}+1\right)
$$


and thus by Gronwall's inequality we obtain

$$
\sup _{t \in[0, T]}\left(\left\|\varepsilon_{t}\right\|_{L^{2}}^{2}+\left\|v_{x}\right\|_{L^{2}}^{2}+\left\|n_{x}\right\|_{L^{2}}^{2}\right)+\beta \int_{0}^{T}\left\|v_{x x}(x, t)\right\|_{L^{2}}^{2} d t \leq C .
$$

By (1.1), Lemmas 2.1, 2.3, and 2.4, Corollary 2.1, Young's inequality, and (2.12) we obtain

$$
\begin{aligned}
\left\|\varepsilon_{x x}\right\|_{L^{2}} \leq & |\alpha|\|\varepsilon\|_{L^{2}}+\|n \varepsilon\|_{L^{2}}+\left\|\varepsilon_{t}\right\|_{L^{2}}+|\delta|\|\varepsilon\|_{L^{2 p+2}}^{2 p+2} \\
\leq & |\alpha|\|\varepsilon\|_{L^{2}}+\|\varepsilon\|_{L^{\infty}}\|n\|_{L^{2}}+\left\|\varepsilon_{t}\right\|_{L^{2}} \\
& +|\delta|\left(C\left\|\varepsilon_{x}\right\|_{L^{2}}^{\frac{p}{2}}\|\varepsilon\|_{L^{2}}^{\frac{p+4}{2(p+2)}}\right) \\
\leq & |\alpha|\|\varepsilon\|_{L^{2}}+\|\varepsilon\|_{L^{\infty}}\|n\|_{L^{2}}+\left\|\varepsilon_{t}\right\|_{L^{2}}+C\left\|\varepsilon_{x}\right\|_{L^{2}}^{\frac{p}{2}} \\
\leq & C\left(\left\|\varepsilon_{t}\right\|_{L^{2}}+1\right) \\
\leq & C .
\end{aligned}
$$

By (1.3), (2.12), and (2.13) we obtain

$$
\sup _{t \in[0, T]}\left(\left\|\varepsilon_{x x}\right\|_{L^{2}}^{2}+\left\|v_{x}\right\|_{L^{2}}^{2}+\left\|n_{x}\right\|_{L^{2}}^{2}+\left\|\varepsilon_{t}\right\|_{L^{2}}^{2}+\left\|n_{t}\right\|_{L^{2}}^{2}\right)+\beta \int_{0}^{T}\left\|v_{x x}(x, t)\right\|_{L^{2}}^{2} d t \leq C .
$$

The Lemma 2.5 is proved.

Corollary 2.2 Suppose that the conditions of Lemma 2.5 are satisfied. Then we have

$$
\sup _{t \in[0, T]}\left(\left\|\varepsilon_{x}\right\|_{L^{\infty}}+\|v\|_{L^{\infty}}+\|n\|_{L^{\infty}}\right) \leq C .
$$

Proof By Lemmas 2.3 and 2.5 the result of Corollary 2.2 is obvious.

Lemma 2.6 Suppose that the conditions of Lemma 2.5 are satisfied, and assume that (1) $\varepsilon_{0} \in H^{3}[0, L], v_{0} \in H^{2}[0, L], n_{0} \in H^{2}[0, L]$, and (2) $\varphi(v) \in C^{2}(R)$. Then for the solution of problem (1.1)-(1.5), we have

$$
\begin{aligned}
& \sup _{t \in[0, T]}\left(\|\varepsilon\|_{H^{3}}+\|v\|_{H^{2}}+\|n\|_{H^{2}}+\left\|\varepsilon_{t}\right\|_{H^{1}}+\left\|v_{t}\right\|_{L^{2}}+\left\|n_{t}\right\|_{H^{1}}\right) \\
& \quad+\beta \int_{0}^{T}\left\|v_{x x x}(x, t)\right\|_{L^{2}}^{2} d t \leq C .
\end{aligned}
$$

Proof Taking the inner product of (2.7) and $-\varepsilon_{t x x}$, it follows that

$$
\left(i \varepsilon_{t t}+\varepsilon_{x x t}+\alpha \varepsilon_{t}-n_{t} \varepsilon-n \varepsilon_{t}+\left(\delta|\varepsilon|^{p} \varepsilon\right)_{t},-\varepsilon_{t x x}\right)=0 .
$$

Since

$$
\operatorname{Im}\left(i \varepsilon_{t t},-\varepsilon_{t x x}\right)=\frac{1}{2} \frac{d}{d t}\left\|\varepsilon_{t x}\right\|_{L^{2}}^{2}, \quad \operatorname{Im}\left(\varepsilon_{x x t}+\alpha \varepsilon_{t},-\varepsilon_{t x x}\right)=0
$$




$$
\begin{aligned}
\left|\operatorname{Im}\left(n_{t} \varepsilon,-\varepsilon_{t x x}\right)\right|= & \left|\operatorname{Im} \int_{0}^{L}\left(n_{t} \varepsilon\right)_{x} \bar{\varepsilon}_{t x} d x\right| \\
= & \left|\operatorname{Im} \int_{0}^{L}\left(n_{t x} \varepsilon+n_{t} \varepsilon_{x}\right) \bar{\varepsilon}_{t x} d x\right| \\
\leq & C\left(\|\varepsilon\|_{L^{\infty}}\left\|n_{t x}\right\|_{L^{2}}+\left\|\varepsilon_{x}\right\|_{L^{\infty}}\left\|n_{t}\right\|_{L^{2}}\right)\left\|\varepsilon_{t x}\right\|_{L^{2}} \\
\leq & C\left(\left\|\varepsilon_{t x}\right\|_{L^{2}}^{2}+\left\|n_{t x}\right\|_{L^{2}}^{2}\right) \\
\leq & C\left(\left\|\varepsilon_{t x}\right\|_{L^{2}}^{2}+\left\|v_{x x}\right\|_{L^{2}}^{2}\right) \\
\left|\operatorname{Im}\left(-n \varepsilon_{t},-\varepsilon_{t x x}\right)\right|= & \left|\operatorname{Im} \int_{0}^{L}\left(n \varepsilon_{t}\right)_{x} \bar{\varepsilon}_{t x} d x\right| \\
= & \left|\operatorname{Im} \int_{0}^{L}\left(n_{x} \varepsilon_{t}+n \varepsilon_{t x}\right) \bar{\varepsilon}_{t x} d x\right| \\
\leq & C\left(\left\|\varepsilon_{t}\right\|_{L^{\infty}}\left\|n_{x}\right\|_{L^{2}}\left\|\varepsilon_{t x}\right\|_{L^{2}}+\|n\|_{L^{\infty}}\left\|\varepsilon_{t x}\right\|_{L^{2}}^{2}\right) \\
\leq & C\left(\left\|\varepsilon_{t x}\right\|_{L^{2}}^{2}+1\right), \\
\operatorname{Im}\left(\left(\delta|\varepsilon|^{p} \varepsilon\right)_{t^{\prime}},-\varepsilon_{t x x}\right) & =-\operatorname{Im} \int_{0}^{L}\left[\left(1+\frac{p}{2}\right) \delta|\varepsilon|^{p} \varepsilon_{t}+\frac{p \delta}{2}|\varepsilon|^{p-2} \varepsilon^{2} \bar{\varepsilon}_{t}\right] \bar{\varepsilon}_{t x x} d x \\
& =\operatorname{Im} \int_{0}^{L}\left[\left(1+\frac{p}{2}\right) \delta|\varepsilon|^{p} \varepsilon_{t}+\frac{p \delta}{2}|\varepsilon|^{p-2} \varepsilon^{2} \bar{\varepsilon}_{t}\right] \bar{\varepsilon}_{t x} d x \\
& \leq C\left(\left\|\varepsilon_{t x}\right\|_{L^{2}}^{2}+1\right),
\end{aligned}
$$

from (2.14) we get

$$
\frac{d}{d t}\left\|\varepsilon_{t x}\right\|_{L^{2}}^{2} \leq C\left(\left\|\varepsilon_{t x}\right\|_{L^{2}}^{2}+\left\|v_{x x}\right\|_{L^{2}}^{2}\right)
$$

Taking the inner product of (1.2) and $v_{x x x x}$, it follows that

$$
\left(v_{t}+\left[\varphi(v)-\beta v_{x}+n+|\varepsilon|^{2}\right]_{x^{\prime}}, v_{x^{4}}\right)=0 .
$$

Since

$$
\begin{aligned}
\left(v_{t}, v_{x^{4}}\right)=\frac{1}{2} \frac{d}{d t} & \left\|v_{x x}\right\|_{L^{2}}^{2}, \quad\left(-\beta v_{x x}, v_{x^{4}}\right)=\beta\left\|v_{x x x}\right\|_{L^{2}}^{2}, \\
\left|\left([\varphi(v)]_{x^{\prime}}, v_{x^{4}}\right)\right| & =\left|\int_{0}^{L}[\varphi(v)]_{x} v_{x^{4}} d x\right| \\
& =\left|\int_{0}^{L}[\varphi(v)]_{x x} v_{x x x} d x\right| \\
& =\left|\int_{0}^{L}\left[\varphi^{\prime \prime}(v) v_{x}^{2}+\varphi^{\prime}(v) v_{x x}\right] v_{x x x} d x\right| \\
& \leq C\left(\left\|v_{x}\right\|_{L^{4}}^{2}+\left\|v_{x x}\right\|_{L^{2}}\right)\left\|v_{x x x}\right\|_{L^{2}} \\
& \leq C\left(\left\|v_{x}\right\|_{L^{2}}^{\frac{3}{2}}\left\|v_{x x}\right\|_{L^{2}}^{\frac{1}{2}}+\left\|v_{x x}\right\|_{L^{2}}\right)\left\|v_{x x x}\right\|_{L^{2}} \\
& \leq \frac{\beta}{4}\left\|v_{x x x}\right\|_{L^{2}}^{2}+C\left(\left\|v_{x x}\right\|_{L^{2}}^{2}+1\right),
\end{aligned}
$$




$$
\begin{aligned}
\left(n_{x}, v_{x^{4}}\right)= & -\int_{0}^{L} n_{x x} v_{x x x} d x=\int_{0}^{L} n_{x x} n_{x x t} d x=\frac{1}{2} \frac{d}{d t}\left\|n_{x x}\right\|_{L^{2}}^{2}, \\
\left|\left(|\varepsilon|_{x}^{2}, v_{x^{4}}\right)\right| & =\left.\left|\int_{0}^{L}\right| \varepsilon\right|_{x x} ^{2} v_{x x x} d x \mid \\
& \leq 2 \int_{0}^{L}\left|\varepsilon\left\|\varepsilon_{x x}\right\| v_{x x x}\right| d x+2 \int_{0}^{L}\left|\varepsilon_{x}\right|^{2}\left|v_{x x x}\right| d x \\
& \leq 2\|\varepsilon\|_{L^{\infty}}\left\|\varepsilon_{x x}\right\|_{L^{2}}\left\|v_{x x x}\right\|_{L^{2}}+2\left\|\varepsilon_{x}\right\|_{L^{4}}^{2}\left\|v_{x x x x}\right\|_{L^{2}} \\
& \leq 2\|\varepsilon\|_{L^{\infty}}\left\|\varepsilon_{x x}\right\|_{L^{2}}\left\|v_{x x x}\right\|_{L^{2}}+2\|\varepsilon\|_{L^{2}}^{\frac{3}{4}}\left\|\varepsilon_{x x}\right\|_{L^{2}}^{\frac{5}{4}}\left\|v_{x x x}\right\|_{L^{2}} \\
& \leq \frac{\beta}{4}\left\|v_{x x x}\right\|_{L^{2}}^{2}+C,
\end{aligned}
$$

from (2.16) we get

$$
\frac{d}{d t}\left(\left\|v_{x x}\right\|_{L^{2}}^{2}+\left\|n_{x x}\right\|_{L^{2}}^{2}\right)+\beta\left\|v_{x x x}\right\|_{L^{2}}^{2} \leq C\left(\left\|v_{x x}\right\|_{L^{2}}^{2}+\left\|n_{x x}\right\|_{L^{2}}^{2}+1\right) .
$$

By (2.15) and (2.17) we obtain

$$
\begin{aligned}
& \frac{d}{d t}\left(\left\|\varepsilon_{t x}\right\|_{L^{2}}^{2}+\left\|v_{x x}\right\|_{L^{2}}^{2}+\left\|n_{x x}\right\|_{L^{2}}^{2}\right)+\beta\left\|v_{x x x}\right\|_{L^{2}}^{2} \\
& \quad \leq C\left(\left\|v_{x x}\right\|_{L^{2}}^{2}+\left\|\varepsilon_{t x}\right\|_{L^{2}}^{2}+\left\|n_{x x}\right\|_{L^{2}}^{2}+1\right),
\end{aligned}
$$

and thus by Gronwall's inequality we obtain

$$
\sup _{t \in[0, T]}\left(\left\|\varepsilon_{t x}\right\|_{L^{2}}^{2}+\left\|v_{x x}\right\|_{L^{2}}^{2}+\left\|n_{x x}\right\|_{L^{2}}^{2}\right)+\beta \int_{0}^{T}\left\|v_{x x x}(x, t)\right\|_{L^{2}}^{2} d t \leq C .
$$

By (1.1), Young's inequality, and (2.18) we obtain

$$
\begin{aligned}
\left\|\varepsilon_{x x x}\right\|_{L^{2}} \leq & |\alpha|\left\|\varepsilon_{x}\right\|_{L^{2}}+\left\|(n \varepsilon)_{x}\right\|_{L^{2}}+\left\|\varepsilon_{t x}\right\|_{L^{2}}+\left\|\delta\left(|\varepsilon|^{p} \varepsilon\right)_{x}\right\|_{L^{2}} \\
\leq & |\alpha|\left\|\varepsilon_{x}\right\|_{L^{2}}+\left(\left\|n_{x} \varepsilon\right\|_{L^{2}}+\left\|n \varepsilon_{x}\right\|_{L^{2}}\right)+\left\|\varepsilon_{t x}\right\|_{L^{2}} \\
& +\left\|\left(1+\frac{p}{2}\right) \delta|\varepsilon|^{p} \varepsilon_{x}+\frac{p \delta}{2}|\varepsilon|^{p-2} \varepsilon^{2} \bar{\varepsilon}_{x}\right\|_{L^{2}} \\
\leq & |\alpha|\left\|\varepsilon_{x}\right\|_{L^{2}}+\left(\left\|n_{x} \varepsilon\right\|_{L^{2}}+\left\|n \varepsilon_{x}\right\|_{L^{2}}\right)+\left\|\varepsilon_{t x}\right\|_{L^{2}} \\
& +\left(1+\frac{p}{2}\right)|\delta|\left\||\varepsilon|^{p} \varepsilon_{x}\right\|_{L^{2}}+\frac{p|\delta|}{2}\left\||\varepsilon|^{p} \varepsilon_{x}\right\|_{L^{2}} \\
\leq & \left\|\varepsilon_{t x}\right\|_{L^{2}}+C \\
\leq & C .
\end{aligned}
$$

By (1.3), (2.18), and (2.19) we obtain

$$
\begin{aligned}
& \sup _{t \in[0, T]}\left(\left\|\varepsilon_{x x x}\right\|_{L^{2}}^{2}+\left\|v_{x x}\right\|_{L^{2}}^{2}+\left\|n_{x x}\right\|_{L^{2}}^{2}+\left\|\varepsilon_{t x}\right\|_{L^{2}}^{2}+\left\|n_{t x}\right\|_{L^{2}}^{2}\right) \\
& \quad+\beta \int_{0}^{T}\left\|v_{x x x}(x, t)\right\|_{L^{2}}^{2} d t \leq C .
\end{aligned}
$$


By (1.2) we obtain

$$
\begin{aligned}
\left\|v_{t}\right\|_{L^{2}} \leq & C\left\|[\varphi(v)]_{x}\right\|_{L^{2}}+\beta\left\|v_{x x}\right\|_{L^{2}}+\left\|n_{x}\right\|_{L^{2}}+\left\||\varepsilon|_{x x}\right\|_{L^{2}} \\
\leq & C\left\|\varphi^{\prime}(v) v_{x}\right\|_{L^{2}}+\beta\left\|v_{x x}\right\|_{L^{2}}+\left\|n_{x}\right\|_{L^{2}} \\
& +\left\|\varepsilon_{x x} \bar{\varepsilon}+2 \varepsilon \bar{\varepsilon}_{x}+\varepsilon \bar{\varepsilon}_{x x}\right\|_{L^{2}} \\
\leq & C\left\|v_{x}\right\|_{L^{2}}+\beta\left\|v_{x x}\right\|_{L^{2}}+\left\|n_{x}\right\|_{L^{2}} \\
& +2\|\varepsilon\|_{L^{\infty}}\left\|\varepsilon_{x x}\right\|_{L^{2}}+2\|\varepsilon\|_{L^{\infty}}\left\|\varepsilon_{x}\right\|_{L^{2}} \\
\leq & C .
\end{aligned}
$$

By (2.20) and (2.21) we obtain

$$
\begin{aligned}
& \sup _{t \in[0, T]}\left(\|\varepsilon\|_{H^{3}}+\|v\|_{H^{2}}+\|n\|_{H^{2}}+\left\|\varepsilon_{t}\right\|_{H^{1}}+\left\|v_{t}\right\|_{L^{2}}+\left\|n_{t}\right\|_{H^{1}}\right) \\
& \quad+\beta \int_{0}^{T}\left\|v_{x x x}(x, t)\right\|_{L^{2}}^{2} d t \leq C .
\end{aligned}
$$

Corollary 2.3 Suppose that the conditions of Lemma 2.6 are satisfied. Then we have

$$
\sup _{t \in[0, T]}\left(\left\|E_{x x}\right\|_{L^{\infty}}+\left\|v_{x}\right\|_{L^{\infty}}+\left\|n_{x}\right\|_{L^{\infty}}+\left\|\varepsilon_{t}\right\|_{L^{\infty}}+\left\|n_{t}\right\|_{L^{\infty}}\right) \leq C .
$$

Proof By Lemmas 2.3 and 2.6 the result of Corollary 2.3 is obvious.

Lemma 2.7 Suppose that (1) $\varepsilon_{0} \in H^{l+2}[0, L], v_{0} \in H^{l+1}[0, L], n_{0} \in H^{l+1}[0, L], l \in Z^{+}$, (2) $\varphi(v) \in C^{l+1}(R),\left|\varphi^{\prime}(v)\right| \leq C\left(|v|^{q}+1\right), 0 \leq q \leq 2$, and (3) $0<p<4$. Then for the solution of problem (1.1)-(1.5), we have

$$
\begin{aligned}
& \sup _{t \in[0, T]}\left(\|\varepsilon\|_{H^{l+2}}+\|v\|_{H^{l+1}}+\|n\|_{H^{l+1}}+\left\|\varepsilon_{t}\right\|_{H^{l}}+\left\|v_{t}\right\|_{H^{l-1}}+\left\|n_{t}\right\|_{H^{l}}\right) \\
& \quad+\beta \int_{0}^{T}\left\|v_{x^{l+2}}(x, t)\right\|_{L^{2}}^{2} d t \leq C .
\end{aligned}
$$

Proof We prove this lemma by mathematical induction. By Lemma 2.6 the lemma is true for $l=1$. Suppose it is is true for $l=k(k \geq 1)$, that is,

$$
\begin{aligned}
& \sup _{t \in[0, T]}\left(\|\varepsilon\|_{H^{k+2}}+\|v\|_{H^{k+1}}+\|n\|_{H^{k+1}}+\left\|\varepsilon_{t}\right\|_{H^{k}}+\left\|v_{t}\right\|_{H^{k-1}}+\left\|n_{t}\right\|_{H^{k}}\right) \\
& \quad+\beta \int_{0}^{T}\left\|v_{x^{k+2}}(x, t)\right\|_{L^{2}}^{2} d t \leq C
\end{aligned}
$$

Next, we will show that the lemma is true for $l=k+1$.

Taking the inner product of (1.2) and $(-1)^{k+2} v_{x^{2 k+4}}$, it follows that

$$
\left(v_{t}+\left[\varphi(v)-\beta v_{x}+n+|\varepsilon|^{2}\right]_{x},(-1)^{k+2} v_{x^{2 k+4}}\right)=0 .
$$

Since

$$
\left(v_{t},(-1)^{k+2} v_{x^{2 k+4}}\right)=\frac{1}{2} \frac{d}{d t}\left\|v_{x^{k+2}}\right\|_{L^{2}}^{2},
$$




$$
\begin{gathered}
\begin{aligned}
\left|\left([\varphi(v)]_{x^{\prime}}(-1)^{k+2} v_{x^{2 k+4}}\right)\right| & =\left|\left([\varphi(v)]_{x^{k+2}},(-1)^{k+2} v_{x^{k+3}}\right)\right| \\
& =\left|\int_{0}^{L}[\varphi(v)]_{x^{k+2}} v_{x^{k+3}} d x\right| \\
& \leq \frac{\beta}{4}\left\|v_{x^{k+3}}\right\|_{L^{2}}^{2}+C\left(\left\|v_{x^{k+2}}\right\|_{L^{2}}^{2}+1\right), \\
\left(-\beta v_{x x},(-1)^{k+2} v_{x^{2 k+4}}\right) & =\beta\left\|v_{x^{k+3}}\right\|_{L^{2}}^{2}, \\
\left(n_{x},(-1)^{k+2} v_{x^{2 k+4}}\right)= & -\int_{0}^{2 L} n_{x^{k+2}} v_{x^{k+3}} d x \\
= & \int_{0}^{L} n_{x^{k+2}} n_{t x^{k+2}} d x \\
\leq & \frac{1}{2} \frac{d}{d t}\left\|n_{x^{k+2}}\right\|_{L^{2}}^{2}, \\
\left|\left(|\varepsilon|_{x^{2}}^{2},(-1)^{k+2} v_{x^{2 k+4}}\right)\right| & =\left|\int_{0}^{L}\left(|\varepsilon|^{2}\right)_{x^{k+2}} v_{x^{k+3}} d x\right| \\
\leq & \frac{\beta}{4}\left\|v_{x^{k+3}}\right\|_{L^{2}}^{2}+C,
\end{aligned}
\end{gathered}
$$

from (2.22) we get

$$
\frac{d}{d t}\left(\left\|v_{x^{k+2}}\right\|_{L^{2}}^{2}+\left\|n_{x^{k+2}}\right\|_{L^{2}}^{2}\right)+\beta\left\|v_{x^{k+3}}\right\|_{L^{2}}^{2} \leq C\left(\left\|v_{x^{k+2}}\right\|_{L^{2}}^{2}+\left\|n_{x^{k+2}}\right\|_{L^{2}}^{2}+1\right) .
$$

By Gronwall's inequality we obtain

$$
\sup _{t \in[0, T]}\left(\left\|v_{x^{k+2}}\right\|_{L^{2}}^{2}+\left\|n_{x^{k+2}}\right\|_{L^{2}}^{2}\right)+\beta \int_{0}^{T}\left\|v_{x^{k+3}}(x, t)\right\|_{L^{2}}^{2} d t \leq C,
$$

and by Eqs. (1.2) and (1.3) we get

$$
\sup _{t \in[0, T]}\left(\left\|v_{t x^{k}}\right\|_{L^{2}}^{2}+\left\|n_{t x^{k+1}}\right\|_{L^{2}}^{2}\right) \leq C .
$$

Taking the inner product of $(2.7)$ and $(-1)^{k+1} \varepsilon_{t}^{2(k+1)}$, it follows that

$$
\left(i \varepsilon_{t t}+\varepsilon_{x x t}+\alpha \varepsilon_{t}-n_{t} \varepsilon-n \varepsilon_{t}+\left(\delta|\varepsilon|^{p} \varepsilon\right)_{t^{\prime}}(-1)^{k+1} \varepsilon_{t x^{2 k+2}}\right)=0 .
$$

Since

$$
\begin{aligned}
& \operatorname{Im}\left(i \varepsilon_{t t},(-1)^{k+1} \varepsilon_{t x^{2 k+2}}\right)= \frac{1}{2} \frac{d}{d t}\left\|\varepsilon_{t x^{k+1}}\right\|_{L^{2}}^{2}, \\
& \operatorname{Im}\left(\varepsilon_{x x t}+\alpha \varepsilon_{t},(-1)^{k+1} \varepsilon_{t x^{2 k+2}}\right)=0, \\
&\left|\operatorname{Im}\left(n_{t} \varepsilon,(-1)^{k+1} \varepsilon_{t x^{2 k+2}}\right)\right|=\left|\operatorname{Im} \int_{0}^{L}\left(n_{t} \varepsilon\right)_{x^{k+1}} \bar{\varepsilon}_{t x^{k+3}} d x\right| \\
& \leq C\left(\left\|\varepsilon_{t x^{k+1}}\right\|_{L^{2}}^{2}+1\right), \\
&\left|\operatorname{Im}\left(-n \varepsilon_{t},(-1)^{k+1} \varepsilon_{t x^{2 k+2}}\right)\right|=\left|\operatorname{Im} \int_{0}^{L}\left(n \varepsilon_{t}\right)_{x^{k+1}} \bar{\varepsilon}_{t x^{k+1}} d x\right| \\
& \leq C\left(\left\|\varepsilon_{x^{k+1}}\right\|_{L^{2}}^{2}+1\right),
\end{aligned}
$$




$$
\begin{aligned}
\left|\operatorname{Im}\left(\left(\delta|\varepsilon|^{p} \varepsilon\right)_{t^{\prime}}(-1)^{k+1} \varepsilon_{t x^{2 k+2}}\right)\right| & =\operatorname{Im} \int_{0}^{L}\left[\left(\delta|\varepsilon|^{p} \varepsilon\right)_{t}\right]_{x^{k+1}} \bar{\varepsilon}_{t x^{k+1}} d x \\
& \leq C\left(\left\|\varepsilon_{t x^{k+1}}\right\|_{L^{2}}^{2}+1\right)
\end{aligned}
$$

from (2.25) we get

$$
\frac{d}{d t}\left\|\varepsilon_{t x^{k+1}}\right\|_{L^{2}}^{2} \leq C\left(\left\|\varepsilon_{t x^{k+1}}\right\|_{L^{2}}^{2}+1\right)
$$

By Gronwall's inequality we obtain

$$
\sup _{t \in[0, T]}\left\|\varepsilon_{t x^{k+1}}\right\|_{L^{2}}^{2} \leq C
$$

and by Eq. (1.1) we get

$$
\sup _{t \in[0, T]}\left\|\varepsilon_{x^{k+3}}\right\|_{L^{2}}^{2} \leq C .
$$

By (2.23), (2.24), (2.26), and (2.27) we get

$$
\begin{aligned}
& \sup _{t \in[0, T]}\left(\|\varepsilon\|_{H^{k+3}}+\|v\|_{H^{k+2}}+\|n\|_{H^{k+2}}+\left\|\varepsilon_{t}\right\|_{H^{k+1}}+\left\|v_{t}\right\|_{H^{k}}+\left\|n_{t}\right\|_{H^{k+1}}\right) \\
& \quad+\beta \int_{0}^{T}\left\|v_{x^{k+3}}(x, t)\right\|_{L^{2}}^{2} d t \leq C .
\end{aligned}
$$

\section{The existence and uniqueness of global generalized solutions for problem}

\section{(1.1)-(1.5)}

Definition 1 The set of functions $\varepsilon(x, t) \in L^{\infty}\left(0, T ; H^{3}[0, L]\right) \cap W^{1, \infty}\left(0, T ; H^{1}[0, L]\right)$, $v(x, t) \in L^{\infty}\left(0, T ; H^{2}[0, L]\right) \cap L^{2}\left(0, T ; H^{3}[0, L]\right) \cap W^{1, \infty}\left(0, T ; L^{2}[0, L]\right)$, and $n(x, t) \in L^{\infty}(0, T$; $\left.H^{2}[0, L]\right) \cap W^{1, \infty}\left(0, T ; H^{1}[0, L]\right)$ is called the generalized solution of problem (1.1)-(1.5) if for any $\omega \in L^{2}[0, L]$, the functions satisfy

$$
\begin{aligned}
& \left(i \varepsilon_{t}, \omega\right)+\left(\varepsilon_{x x}, \omega\right)+(\alpha \varepsilon, \omega)-(n \varepsilon, \omega)+\left(\delta|\varepsilon|^{p} \varepsilon, \omega\right)=0, \\
& \left(v_{t}, \omega\right)+\left([\varphi(v)]_{x}, \omega\right)-\left(\beta v_{x x}, \omega\right)+\left(n_{x}, \omega\right)-\left(|\varepsilon|_{x}^{2}, \omega\right)=0, \\
& \left(n_{t}, \omega\right)+\left(v_{x}, \omega\right)=0, \\
& (\varepsilon(x, 0), \omega)=\left(\varepsilon_{0}(x), \omega\right), \quad(v(x, 0), \omega)=\left(v_{0}(x), \omega\right), \\
& (n(x, 0), \omega)=\left(n_{0}(x), \omega\right), \\
& \varepsilon(0, t)=\varepsilon(L, t)=v(0, t)=v(L, t)=n(0, t)=n(L, t)=0 .
\end{aligned}
$$

Theorem 3.1 Suppose that the conditions of Lemma 2.6 are satisfied. Then there exists a global generalized solution of the initial boundary value problem (1.1)-(1.5),

$$
\begin{aligned}
& \varepsilon(x, t) \in L^{\infty}\left(0, T ; H^{3}[0, L]\right), \quad \varepsilon_{t}(x, t) \in L^{\infty}\left(0, T ; H^{1}[0, L]\right), \\
& v(x, t) \in L^{\infty}\left(0, T ; H^{2}[0, L]\right) \cap L^{2}\left(0, T ; H^{3}[0, L]\right), \quad v_{t}(x, t) \in L^{\infty}\left(0, T ; L^{2}[0, L]\right), \\
& n(x, t) \in L^{\infty}\left(0, T ; H^{2}[0, L]\right), \quad n_{t}(x, t) \in L^{\infty}\left(0, T ; H^{1}[0, L]\right) .
\end{aligned}
$$


Proof By using the Galerkin method we choose a basis $\left\{\omega_{j}(x)\right\} \subseteq H^{2}[0, L] \cap H_{0}^{1}[0, L]$ consisting of the eigenfunctions of the problem

$$
\begin{aligned}
& -\triangle \omega_{j}(x)=\lambda_{j} \omega_{j}(x), \quad j=1,2, \ldots, m, \\
& \left.\omega_{j}(x)\right|_{x=0}=\left.\omega_{j}(x)\right|_{x=L}=0 .
\end{aligned}
$$

Then the approximate solution of problem (1.1)-(1.4) can be written as

$$
\begin{aligned}
& \varepsilon_{m}(x, t)=\sum_{j=1}^{m} \alpha_{j m}(t) \omega_{j}(x), \quad v_{m}(x, t)=\sum_{j=1}^{m} \beta_{j m}(t) \omega_{j}(x), \\
& n_{m}(x, t)=\sum_{j=1}^{m} \gamma_{j m}(t) \omega_{j}(x) .
\end{aligned}
$$

According to Galerkin's method, the undetermined coefficients $\alpha_{j m}(t), \beta_{j m}(t)$, and $\gamma_{j m}(t)$ need to satisfy the following initial value problem of ordinary differential equations:

$$
\begin{aligned}
& \left(i \varepsilon_{m t}+\varepsilon_{m x x}+\left(\alpha-n_{m}\right) \varepsilon_{m}+\delta\left|\varepsilon_{m}\right|^{p} \varepsilon_{m}, \omega\right)=0, \\
& \left(v_{m t}+\left[\varphi\left(v_{m}\right)\right]_{x}-\beta v_{m x x}+n_{m x}-|\varepsilon|_{m x}^{2}, \omega\right)=0, \\
& \left(n_{m t}+v_{m x}, \omega\right)=0, \\
& \varepsilon_{m}(x, 0)=\varepsilon_{m 0}(x), \quad v_{m}(x, 0)=v_{m 0}(x), \quad n_{m}(x, 0)=n_{m 0}(x), \quad x \in[0, L],
\end{aligned}
$$

where

$$
\begin{aligned}
& \varepsilon_{m 0}(x) \rightarrow \varepsilon_{0}(x) \quad \text { in } H^{3}[0, L], \quad v_{m 0}(x) \rightarrow v_{0}(x) \quad \text { in } H^{2}[0, L], \\
& n_{m 0}(x) \rightarrow n_{0}(x) \quad \text { in } H^{2}[0, L], m \rightarrow \infty .
\end{aligned}
$$

Similarly to the proof of Lemmas 2.1, 2.4, 2.5, and 2.6, for the solution $\varepsilon_{m}(x, t), v_{m}(x, t)$, $n_{m}(x, t)$ of problem (3.9)-(3.12), we can establish the following estimate:

$$
\begin{aligned}
& \sup _{t \in[0, T]}\left(\left\|\varepsilon_{m}\right\|_{H^{3}}+\left\|v_{m}\right\|_{H^{2}}+\left\|n_{m}\right\|_{H^{2}}+\left\|\varepsilon_{m t}\right\|_{H^{1}}+\left\|v_{m t}\right\|_{L^{2}}+\left\|n_{m t}\right\|_{H^{1}}\right) \\
& \quad+\beta \int_{0}^{T}\left\|v_{m}\right\|_{H^{3}}^{2} d t \leq C,
\end{aligned}
$$

where the constant $C$ is independent of $m$. By compact argument we can choose a subsequence $\varepsilon_{v}(x, t), v_{v}(x, t), n_{v}(x, t)$ such that, as $v \rightarrow \infty$,

$$
\begin{aligned}
& \varepsilon_{v}(x, t) \rightarrow \varepsilon(x, t) \quad \text { in } L^{\infty}\left(0, T ; H^{3}[0, L]\right) \text { weakly star, } \\
& \varepsilon_{v}(x, t) \rightarrow \varepsilon(x, t) \text { in strong topology of } L^{2}\left(Q_{T}\right), \\
& \varepsilon_{v t}(x, t) \rightarrow \varepsilon_{t}(x, t) \quad \text { in } L^{\infty}\left(0, T ; H^{1}[0, L]\right) \text { weakly star, } \\
& v_{v}(x, t) \rightarrow v(x, t) \text { in } L^{\infty}\left(0, T ; H^{2}[0, L]\right) \cap L^{2}\left(0, T ; H^{3}[0, L]\right) \text { weakly star, } \\
& v_{v}(x, t) \rightarrow v(x, t) \text { in strong topology of } L^{2}\left(Q_{T}\right),
\end{aligned}
$$




$$
\begin{aligned}
& v_{v t}(x, t) \rightarrow v_{t}(x, t) \quad \text { in } L^{\infty}\left(0, T ; L^{2}[0, L]\right) \text { weakly star, } \\
& n_{v}(x, t) \rightarrow n(x, t) \quad \text { in } L^{\infty}\left(0, T ; H^{2}[0, L]\right) \text { weakly star, } \\
& n_{v}(x, t) \rightarrow n(x, t) \quad \text { in strong topology of } L^{2}\left(Q_{T}\right), \\
& n_{v t}(x, t) \rightarrow n_{t}(x, t) \quad \text { in } L^{\infty}\left(0, T ; H^{1}[0, L]\right) \text { weakly star, } \\
& \left|\varepsilon_{v}\right|^{p} \varepsilon_{v} \rightarrow|\varepsilon|^{p} \varepsilon \quad \text { in } L^{\infty}\left(0, T ; L^{2}[0, L]\right) \text { weakly star, } \\
& \left|\varepsilon_{v}\right|_{x}^{2} \rightarrow|\varepsilon|_{x}^{2} \quad \text { in } L^{\infty}\left(0, T ; L^{2}[0, L]\right) \text { weakly star, } \\
& n_{v} \varepsilon_{v} \rightarrow n \varepsilon \quad \text { in } L^{\infty}\left(0, T ; L^{2}[0, L]\right) \text { weakly star, } \\
& \varphi\left(v_{v}\right) \rightarrow \varphi(v) \quad \text { in } L^{\infty}\left(0, T ; L^{2}[0, L]\right) \text { weakly star, }
\end{aligned}
$$

where $Q_{T}=[0, L] \times[0, T]$. Hence, taking $m=v \rightarrow \infty$ in (3.9)-(3.13), by using the density of $\omega_{j}(x)$ in $L^{2}[0, L]$ we get the existence of a local generalized solution for problem (1.1)(1.5). From the conditions of the theorem and a priori estimates in Sect. 2 we can get the existence of a global generalized solution for problem (1.1)-(1.5) by the continuation extension principle.

Theorem 3.2 Suppose that the conditions of Theorem 3.1 are satisfied. Then the global generalized solution of the initial boundary value problem (1.1)-(1.5) is unique, and

$$
\begin{aligned}
& \varepsilon(x, t) \in L^{\infty}\left(0, T ; H^{3}[0, L]\right), \quad E_{t}(x, t) \in L^{\infty}\left(0, T ; H^{1}[0, L]\right), \\
& v(x, t) \in L^{\infty}\left(0, T ; H^{2}[0, L]\right) \cap L^{2}\left(0, T ; H^{3}[0, L]\right), \quad v_{t}(x, t) \in L^{\infty}\left(0, T ; L^{2}[0, L]\right), \\
& n(x, t) \in L^{\infty}\left(0, T ; H^{2}[0, L]\right), \quad n_{t}(x, t) \in L^{\infty}\left(0, T ; H^{1}[0, L]\right) .
\end{aligned}
$$

Proof Suppose that there are two solutions $\varepsilon_{1}, n_{1}, \varphi_{1}$ and $\varepsilon_{2}, n_{2}, \varphi_{2}$. Let

$$
\varepsilon=\varepsilon_{1}-\varepsilon_{2}, \quad v=v_{1}-v_{2}, \quad n=n_{1}-n_{2} .
$$

From (1.1)-(1.5) we get

$$
\begin{aligned}
& i \varepsilon_{t}+\varepsilon_{x x}+\alpha \varepsilon-n_{1} \varepsilon_{1}+n_{2} \varepsilon_{2}+\delta\left|\varepsilon_{1}\right|^{p} \varepsilon_{1}-\delta\left|\varepsilon_{2}\right|^{p} \varepsilon_{2}=0, \\
& v_{t}+\left[\varphi\left(v_{1}\right)\right]_{x}-\left[\varphi\left(v_{2}\right)\right]_{x}-\beta v_{x x}+n_{x}+\left|\varepsilon_{1}\right|_{x}^{2}-\left|\varepsilon_{2}\right|_{x}^{2}=0, \\
& n_{t}+v_{x}=0
\end{aligned}
$$

with initial data

$$
\left.\varepsilon\right|_{t=0}=0,\left.\quad v\right|_{t=0}=0,\left.\quad n\right|_{t=0}=0
$$

and boundary conditions

$$
\varepsilon(0, t)=\varepsilon(L, t)=v(0, t)=v(L, t)=n(0, t)=n(L, t)=0 .
$$

Taking the inner product of (3.14) and $\varepsilon$, it follows that

$$
\left(i \varepsilon_{t}+\varepsilon_{x x}+\alpha \varepsilon-n_{1} \varepsilon_{1}+n_{2} \varepsilon_{2}+\delta\left|\varepsilon_{1}\right|^{p} \varepsilon_{1}-\delta\left|\varepsilon_{2}\right|^{p} \varepsilon_{2}, \varepsilon\right)=0 .
$$


Since

$$
\begin{aligned}
\operatorname{Im}\left(i \varepsilon_{t}, \varepsilon\right)=\frac{1}{2} \frac{d}{d t}\|\varepsilon\|_{L^{2}}^{2}, & \operatorname{Im}\left(\varepsilon_{x x}+\alpha \varepsilon, \varepsilon\right)=0, \\
\left|\operatorname{Im}\left(n_{1} \varepsilon_{1}-n_{2} \varepsilon_{2}, \varepsilon\right)\right| & =\left|\operatorname{Im} \int_{0}^{L} n \varepsilon_{1} \bar{\varepsilon} d x\right| \\
& \leq \frac{1}{2}\left\|\varepsilon_{1}\right\|_{L^{\infty}}\left(\|n\|_{L^{2}}^{2}+\|\varepsilon\|_{L^{2}}^{2}\right) \\
& \leq C\left(\|n\|_{L^{2}}^{2}+\|\varepsilon\|_{L^{2}}^{2}\right) .
\end{aligned}
$$

By the Lagrange mean value theorem we get

$$
\begin{aligned}
\left.|| \varepsilon_{1}\right|^{p} \varepsilon_{1}-\left|\varepsilon_{2}\right|^{p} \varepsilon_{2} \mid & =\left.|| \varepsilon_{1}\right|^{p} \varepsilon_{1}-\left|\varepsilon_{1}\right|^{p} \varepsilon_{2}+\left|\varepsilon_{1}\right|^{p} \varepsilon_{2}-\left|\varepsilon_{2}\right|^{p} \varepsilon_{2} \mid \\
& \leq\left|\varepsilon_{1}\right|^{p}\left|\varepsilon_{1}-\varepsilon_{2}\right|+\left|\varepsilon_{2}\right|\left(\left|\varepsilon_{1}\right|^{p}-\left|\varepsilon_{2}\right|^{p}\right) \\
& \leq\left|\varepsilon_{1}\right|^{p}|\varepsilon|+p\left|\varepsilon_{2}\right| \sup _{t \in[0, T]}\left(\left|\varepsilon_{1}\right|^{p-1},\left|\varepsilon_{2}\right|^{p-1}\right)|\varepsilon| \\
& \leq(p+1) \sup _{t \in[0, T]}\left(\left|\varepsilon_{1}\right|^{p},\left|\varepsilon_{2}\right|^{p}\right)|\varepsilon| .
\end{aligned}
$$

Therefore

$$
\begin{aligned}
\left|\operatorname{Im}\left(\delta\left(\left|\varepsilon_{1}\right|^{p} \varepsilon_{1}-\left|\varepsilon_{2}\right|^{p} \varepsilon_{2}\right), \varepsilon\right)\right| & \leq\left.|\delta| \int_{0}^{L}|| \varepsilon_{1}\right|^{p} \varepsilon_{1}-\left|\varepsilon_{2}\right|^{p} \varepsilon_{2}|| \varepsilon|d x| \\
& \leq|\delta| \int_{0}^{L}(p+1) \sup _{t \in[0, T]}\left(\left|\varepsilon_{1}\right|^{p},\left|\varepsilon_{1}\right|^{p}\right)|\varepsilon|^{2} d x \\
& \leq|\delta|(p+1) \sup _{t \in[0, T]}\left(\left\|\varepsilon_{1}\right\|_{L^{\infty}}^{p}\left\|\varepsilon_{1}\right\|_{L^{\infty}}^{p}\right)\|\varepsilon\|_{L^{2}}^{2} \\
& \leq C\|\varepsilon\|_{L^{2}}^{2} .
\end{aligned}
$$

Hence from (3.19) we get

$$
\frac{d}{d t}\|\varepsilon\|_{L^{2}}^{2} \leq C\left(\|n\|_{L^{2}}^{2}+\|\varepsilon\|_{L^{2}}^{2}\right)
$$

Taking the inner product of (3.15) and $v$, it follows that

$$
\left(v_{t}+\left[\varphi\left(v_{1}\right)\right]_{x}-\left[\varphi\left(v_{2}\right)\right]_{x}-\beta v_{x x}+n_{x}+\left|\varepsilon_{1}\right|_{x}^{2}-\left|\varepsilon_{2}\right|_{x}^{2}, v\right)=0
$$

Since

$$
\begin{aligned}
&\left(v_{t}, v\right)=\frac{1}{2} \frac{d}{d t}\|v\|_{L^{2}}^{2}, \quad\left(-\beta v_{x x}, v\right)=\beta\left\|v_{x}\right\|_{L^{2}}^{2}, \\
&\left|\left(\left[\varphi\left(v_{1}\right)\right]_{x}-\left[\varphi\left(v_{2}\right)\right]_{x}, v\right)\right|=\left|\left(\varphi^{\prime}(\xi) v, v_{x}\right)\right| \\
& \leq\left\|\varphi^{\prime}(\xi)\right\|_{L^{\infty}}\|v\|_{L^{2}}\left\|v_{x}\right\|_{L^{2}} \\
& \leq C\left(|\xi|^{q}+1\right)\|v\|_{L^{2}}\left\|v_{x}\right\|_{L^{2}} \\
& \leq C\left(\left\|v_{1}\right\|_{L^{\infty}}^{q}+\left\|v_{2}\right\|_{L^{\infty}}^{q}+1\right)\|v\|_{L^{2}}\left\|v_{x}\right\|_{L^{2}} \\
& \leq C\left(\|v\|_{L^{2}}^{2}+\left\|n_{t}\right\|_{L^{2}}^{2}\right),
\end{aligned}
$$




$$
\begin{aligned}
&\left|\left(n_{x}, v\right)\right| \leq \frac{1}{2}\left(\|n\|_{L^{2}}^{2}+\left\|n_{t}\right\|_{L^{2}}^{2}\right), \\
&\left(\left|\varepsilon_{1}\right|_{x}^{2}-\left|\varepsilon_{2}\right|_{x}^{2}, v\right)=\left|\int_{0}^{L}\left(\left|\varepsilon_{1}\right|^{2}-\left|\varepsilon_{2}\right|^{2}\right) v_{x} d x\right| \\
&=\left|\int_{0}^{L}\left(\varepsilon \bar{\varepsilon}_{1}+\varepsilon_{2} \bar{\varepsilon}\right) v_{x} d x\right| \\
& \leq\left(\left\|\varepsilon_{1}\right\|_{L^{\infty}}+\left\|\varepsilon_{2}\right\|_{L^{\infty}}\right)\|\varepsilon\|_{L^{2}}\left\|n_{t}\right\|_{L^{2}} \\
& \leq C\left(\|\varepsilon\|_{L^{2}}^{2}+\left\|n_{t}\right\|_{L^{2}}^{2}\right),
\end{aligned}
$$

from (3.21) we get

$$
\frac{d}{d t}\|v\|_{L^{2}}^{2} \leq C\left(\|\varepsilon\|_{L^{2}}^{2}+\|v\|_{L^{2}}^{2}+\|n\|_{L^{2}}^{2}+\left\|n_{t}\right\|_{L^{2}}^{2}\right)
$$

Since

$$
\frac{d}{d t}\|n\|_{L^{2}}^{2}=\frac{d}{d t} \int_{0}^{L} n^{2} d x \leq\|n\|_{L^{2}}^{2}+\left\|n_{t}\right\|_{L^{2}}^{2}
$$

by (3.20), (3.22), and (3.23) we get

$$
\frac{d}{d t}\left(\|\varepsilon\|_{L^{2}}^{2}+\|v\|_{L^{2}}^{2}+\|n\|_{L^{2}}^{2}\right) \leq C\left(\|\varepsilon\|_{L^{2}}^{2}+\|n\|_{L^{2}}^{2}+\|v\|_{L^{2}}^{2}+\left\|n_{t}\right\|_{L^{2}}^{2}\right) .
$$

Taking the inner product of (3.15) and $-v_{x x}$, it follows that

$$
\left(v_{t}+\left[\varphi\left(v_{1}\right)\right]_{x}-\left[\varphi\left(v_{2}\right)\right]_{x}-\beta v_{x x}+n_{x}+\left|\varepsilon_{1}\right|_{x}^{2}-\left|\varepsilon_{2}\right|_{x}^{2},-v_{x x}\right)=0 .
$$

Since

$$
\begin{aligned}
\left(v_{t},-v_{x x}\right)=\frac{1}{2} \frac{d}{d t}\left\|v_{x}\right\|_{L^{2}}^{2}, \quad\left(-\beta v_{x x},-v_{x x}\right)=\beta\left\|v_{x x}\right\|_{L^{2}}^{2}, & \\
\left|\left(\left[\varphi\left(v_{1}\right)\right]_{x}-\left[\varphi\left(v_{2}\right)\right]_{x^{\prime}},-v_{x x}\right)\right| & =\left|\int_{0}^{L}\left[\varphi\left(v_{1}\right)-\varphi\left(v_{2}\right)\right] v_{x x x} d x\right| \\
& =\left|\left(\varphi^{\prime}(\xi) v, v_{x x x}\right)\right| \\
& =\left\|\varphi^{\prime}(\xi)\right\|_{L^{\infty}}\left|\left(v_{x}, v_{x x}\right)\right| \\
& \leq\left|\varphi^{\prime}(\xi)\right|\left\|v_{x}\right\|_{L^{2}}\left\|v_{x x}\right\|_{L^{2}}^{2} \\
& \leq C\left(|\xi|^{q}+1\right)\left\|v_{x}\right\|_{L^{2}}\left\|v_{x x}\right\|_{L^{2}} \\
& \leq C\left(\left\|v_{1}\right\|_{L^{\infty}}^{q}+\left\|v_{2}\right\|_{L^{\infty}}^{q}+1\right)\left\|v_{x}\right\|_{L^{2}}\left\|v_{x x}\right\|_{L^{2}} \\
& \leq \frac{\beta}{2}\left\|v_{x x}\right\|_{L^{2}}^{2}+C\left\|v_{x}\right\|_{L^{2}}^{2}, \\
\left(n_{x},-v_{x x}\right)=-\int_{0}^{L} n_{x} v_{x x} d x= & \int_{0}^{L} n_{x} n_{x t} d x=\frac{1}{2} \frac{d}{d t}\left\|n_{x}\right\|_{L^{2}}^{2},
\end{aligned}
$$




$$
\begin{aligned}
\left|\left(\left|\varepsilon_{1}\right|_{x}^{2}-\left|\varepsilon_{2}\right|_{x}^{2},-v_{x x}\right)\right|= & \left|\int_{0}^{L}\left(\left|\varepsilon_{1}\right|_{x}^{2}-\left|\varepsilon_{2}\right|_{x}^{2}\right) v_{x x} d x\right| \\
= & \left|\int_{0}^{L}\left(\varepsilon_{x} \bar{\varepsilon}_{1}+\varepsilon_{2 x} \bar{\varepsilon}+\varepsilon \bar{\varepsilon}_{1 x}+\varepsilon_{2} \bar{\varepsilon}_{x}\right) v_{x x} d x\right| \\
= & \left|\int_{0}^{L}\left(\varepsilon_{x} \bar{\varepsilon}_{1}+\varepsilon_{2} \bar{\varepsilon}_{x}\right) v_{x x} d x\right| \\
& +\left|\int_{0}^{L}\left(\varepsilon \bar{\varepsilon}_{1 x}+\varepsilon_{2 x} \bar{\varepsilon}_{x}\right) v_{x x} d x\right| \\
= & \left|\int_{0}^{L}\left(\varepsilon_{x} \bar{\varepsilon}_{1}+\varepsilon_{2} \bar{\varepsilon}_{x}\right)_{x} v_{x} d x\right| \\
& +\left|\int_{0}^{L}\left(\varepsilon \bar{\varepsilon}_{1 x}+\varepsilon_{2 x} \bar{\varepsilon}_{x}\right)_{x} v_{x} d x\right| \\
\leq & \int_{0}^{L}\left(\left|\varepsilon_{1}\right|+\left|\varepsilon_{2}\right|\right)\left|\varepsilon_{x} \| v_{x x}\right| d x \\
& +\int_{0}^{L}\left(\left|\varepsilon_{1 x}\right|+\left|\varepsilon_{2 x}\right|\right)\left|\varepsilon \| v_{x x}\right| d x \\
\leq & \left(\left\|\varepsilon_{1}\right\|_{L^{\infty}}+\left\|\varepsilon_{2}\right\|_{L^{\infty}}\right)\left\|\varepsilon_{x}\right\|_{L^{2}}\left\|v_{x x}\right\|_{L^{2}} \\
& +\left(\left\|\varepsilon_{1 x}\right\|_{L^{\infty}}+\left\|\varepsilon_{2 x}\right\|_{L^{\infty}}\right)\|\varepsilon\|_{L^{2}}\left\|v_{x x}\right\|_{L^{2}} \\
\leq & \left\|v_{x x}\right\|_{L^{2}}^{2}+C\|\varepsilon\|_{L^{2}}^{2},
\end{aligned}
$$

from (1.3) and (3.25) we get

$$
\frac{d}{d t}\left(\left\|v_{x}\right\|_{L^{2}}^{2}+\left\|n_{x}\right\|_{L^{2}}^{2}\right)=\frac{d}{d t}\left(\left\|n_{t}\right\|_{L^{2}}^{2}+\left\|n_{x}\right\|_{L^{2}}^{2}\right) \leq C\left(\|\varepsilon\|_{L^{2}}^{2}+\left\|v_{x}\right\|_{L^{2}}^{2}\right) .
$$

By (3.24) and (3.26) we obtain

$$
\begin{aligned}
& \frac{d}{d t}\left(\|\varepsilon\|_{L^{2}}^{2}+\|v\|_{L^{2}}^{2}+\|n\|_{L^{2}}^{2}+\left\|n_{t}\right\|_{L^{2}}^{2}+\left\|n_{x}\right\|_{L^{2}}^{2}\right) \\
& \quad \leq C\left(\|\varepsilon\|_{L^{2}}^{2}+\|n\|_{L^{2}}^{2}+\|v\|_{L^{2}}^{2}+\left\|n_{t}\right\|_{L^{2}}^{2}+\left\|n_{x}\right\|_{L^{2}}^{2}\right)
\end{aligned}
$$

By using Gronwall's inequality we obtain

$$
\varepsilon \equiv 0, \quad v \equiv 0, \quad n \equiv 0,
$$

and hence

$$
\varepsilon_{1}=\varepsilon_{2}, \quad \nu_{1}=v_{2}, \quad n_{1}=n_{2} .
$$

Therefore the proof of Theorem 3.2 is completed.

4 The regularity of global generalized solution for problem (1.1)-(1.5)

To get the regularity of the global generalized solution for problem (1.1)-(1.5), we need the following lemma and corollary. 
Lemma 4.1 Suppose that the conditions of Lemma 2.6 are satisfied, and assume that (1) $\varepsilon_{0} \in H^{4}[0, L], v_{0} \in H^{3}[0, L], n_{0} \in H^{3}[0, L]$, and (2) $\varphi(v) \in C^{3}(R)$. Then for the solution of problem (1.1)-(1.5), we have

$$
\begin{aligned}
& \sup _{t \in[0, T]}\left(\|\varepsilon\|_{H^{4}}+\|v\|_{H^{3}}+\|n\|_{H^{3}}+\left\|\varepsilon_{t}\right\|_{H^{2}}+\left\|v_{t}\right\|_{H^{1}}+\left\|n_{t}\right\|_{H^{2}}\right) \\
& \quad+\beta \int_{0}^{T}\left\|v_{x^{4}}(x, t)\right\|_{L^{2}}^{2} d t \leq C .
\end{aligned}
$$

Proof Taking the inner product of (2.7) and $-\varepsilon_{t x^{4}}$, it follows that

$$
\left(i \varepsilon_{t t}+\varepsilon_{x x t}+\alpha \varepsilon_{t}-n_{t} \varepsilon-n \varepsilon_{t}+\left(\delta|\varepsilon|^{p} \varepsilon\right)_{t^{\prime}}-\varepsilon_{t x^{4}}\right)=0 .
$$

Since

$$
\begin{aligned}
& \operatorname{Im}\left(i \varepsilon_{t t},-\varepsilon_{t x^{4}}\right)=\frac{1}{2} \frac{d}{d t}\left\|\varepsilon_{t x x}\right\|_{L^{2}}^{2}, \quad \operatorname{Im}\left(\varepsilon_{x x t}+\alpha \varepsilon_{t},-\varepsilon_{t x^{4}}\right)=0, \\
& \left|\operatorname{Im}\left(n_{t} \varepsilon,-\varepsilon_{t x^{4}}\right)\right|=\left|\operatorname{Im} \int_{0}^{L}\left(n_{t} \varepsilon\right)_{x x} \bar{\varepsilon}_{t x x} d x\right| \\
& =\left|\operatorname{Im} \int_{0}^{L}\left(n_{x t} \varepsilon+n_{t} \varepsilon_{x}\right)_{x} \bar{\varepsilon}_{t x x} d x\right| \\
& =\left|\operatorname{Im} \int_{0}^{L}\left(n_{x x t} \varepsilon+n_{x t} \varepsilon_{x}+n_{x t} \varepsilon_{x}+n_{t} \varepsilon_{x x}\right) \bar{\varepsilon}_{t x x} d x\right| \\
& \leq C\left(\left\|\varepsilon_{t x x}\right\|_{L^{2}}^{2}+\left\|v_{x x x}\right\|_{L^{2}}^{2}\right) \text {, } \\
& \left|\operatorname{Im}\left(-n \varepsilon_{t},-\varepsilon_{t x^{4}}\right)\right|=\left|\operatorname{Im} \int_{0}^{L}\left(n \varepsilon_{t}\right)_{x x} \bar{\varepsilon}_{t x x} d x\right| \\
& \leq C\left(\left\|\varepsilon_{t x x}\right\|_{L^{2}}^{2}+1\right) \text {, } \\
& \operatorname{Im}\left(\left(\delta|\varepsilon|^{p} \varepsilon\right)_{t},-\varepsilon_{t x^{4}}\right)=\operatorname{Im} \int_{0}^{L}\left[\left(1+\frac{p}{2}\right) \delta|\varepsilon|^{p} \varepsilon_{t}+\frac{p \delta}{2}|\varepsilon|^{p-2} \varepsilon^{2} \bar{\varepsilon}_{t}\right] \bar{\varepsilon}_{t}^{(4)} d x \\
& =\operatorname{Im} \int_{0}^{L}\left[\left(1+\frac{p}{2}\right) \delta|\varepsilon|^{p} \varepsilon_{t}+\frac{p \delta}{2}|\varepsilon|^{p-2} \varepsilon^{2} \bar{\varepsilon}_{t}\right]_{x x} \bar{\varepsilon}_{t x x} d x \\
& \leq C\left(\left\|\varepsilon_{t x x}\right\|_{L^{2}}^{2}+1\right) \text {, }
\end{aligned}
$$

from (4.1) we get

$$
\frac{d}{d t}\left\|\varepsilon_{t x x}\right\|_{L^{2}}^{2} \leq C\left(\left\|\varepsilon_{t x x}\right\|_{L^{2}}^{2}+\left\|v_{x x x}\right\|_{L^{2}}^{2}\right)
$$

Taking the inner product of (1.2) and $v_{x^{6}}$, it follows that

$$
\left(v_{t}+\left[\varphi(v)-\beta v_{x}+n+|\varepsilon|^{2}\right]_{x^{\prime}}, v_{x^{6}}\right)=0 .
$$

Since

$$
\left(v_{t}, v_{x^{6}}\right)=\frac{1}{2} \frac{d}{d t}\left\|v_{x x x}\right\|_{L^{2}}^{2}, \quad\left(-\beta v_{x x}, v_{x^{6}}\right)=\beta\left\|v_{x^{4}}\right\|_{L^{2}}^{2},
$$




$$
\begin{aligned}
& \begin{aligned}
\left|\left([\varphi(v)]_{x^{\prime}}, v_{x^{6}}\right)\right| & =\left|\int_{0}^{L}[\varphi(v)]_{x} v_{x^{6}} d x\right| \\
& =\left|\int_{0}^{L}[\varphi(v)]_{x x x} v_{x^{4}} d x\right| \\
& \leq \frac{\beta}{4}\left\|v_{x^{4}}\right\|_{L^{2}}^{2}+C\left(\left\|v_{x x x}\right\|_{L^{2}}^{2}+1\right),
\end{aligned} \\
& \left(n_{x}, v_{x^{6}}\right)=-\int_{0}^{L} n_{x x x} v_{x^{4}} d x=\int_{0}^{L} n_{x x x} n_{t x^{3}} d x=\frac{1}{2} \frac{d}{d t}\left\|n_{x x x}\right\|_{L^{2}}^{2}, \\
& \left|\left(|\varepsilon|_{x}^{2}, v_{x^{6}}\right)\right|=\left.\left|\int_{0}^{L}\right| \varepsilon\right|_{x x x} ^{2} v_{x^{4}} d x \mid \leq \frac{\beta}{4}\left\|v_{x^{4}}\right\|_{L^{2}}^{2}+C,
\end{aligned}
$$

from (4.3) we get

$$
\frac{d}{d t}\left(\left\|v_{x x x}\right\|_{L^{2}}^{2}+\left\|n_{x x x}\right\|_{L^{2}}^{2}\right)+\beta\left\|v_{x^{4}}\right\|_{L^{2}}^{2} \leq C\left(\left\|v_{x x x}\right\|_{L^{2}}^{2}+\left\|n_{x x x}\right\|_{L^{2}}^{2}+1\right) .
$$

By (4.2) and (4.4) we obtain

$$
\begin{aligned}
& \frac{d}{d t}\left(\left\|\varepsilon_{t x x}\right\|_{L^{2}}^{2}+\left\|v_{x x x}\right\|_{L^{2}}^{2}+\left\|n_{x x x}\right\|_{L^{2}}^{2}\right)+\beta\left\|v_{x^{4}}\right\|_{L^{2}}^{2} \\
& \quad \leq C\left(\left\|v_{x x x}\right\|_{L^{2}}^{2}+\left\|\varepsilon_{t x x}\right\|_{L^{2}}^{2}+\left\|n_{x x x}\right\|_{L^{2}}^{2}+1\right)
\end{aligned}
$$

and thus by using Gronwall's inequality we obtain

$$
\sup _{t \in[0, T]}\left(\left\|\varepsilon_{t x x}\right\|_{L^{2}}^{2}+\left\|v_{x x x}\right\|_{L^{2}}^{2}+\left\|n_{x x x}\right\|_{L^{2}}^{2}\right)+\beta \int_{0}^{T}\left\|v_{x^{4}}(x, t)\right\|_{L^{2}}^{2} d t \leq C .
$$

By (1.1) and Young's inequality we obtain

$$
\begin{aligned}
\left\|\varepsilon_{x^{4}}\right\|_{L^{2}} & \leq|\alpha|\left\|\varepsilon_{x x}\right\|_{L^{2}}+\left\|(n \varepsilon)_{x x}\right\|_{L^{2}}+\left\|\varepsilon_{t x x}\right\|_{L^{2}}+\left\|\delta\left(|\varepsilon|^{p} \varepsilon\right)_{x x}\right\|_{L^{2}} \\
& \leq C\left(\left\|\varepsilon_{t x x}\right\|_{L^{2}}+1\right) .
\end{aligned}
$$

By (1.3), (4.5), and (4.6) we obtain

$$
\begin{aligned}
& \sup _{t \in[0, T]}\left(\left\|\varepsilon_{x^{4}}\right\|_{L^{2}}^{2}+\left\|v_{x x x}\right\|_{L^{2}}^{2}+\left\|n_{x x x}\right\|_{L^{2}}^{2}+\left\|\varepsilon_{t x x}\right\|_{L^{2}}^{2}+\left\|n_{t x x}\right\|_{L^{2}}^{2}\right) \\
& \quad+\beta \int_{0}^{T}\left\|v_{x^{4}}(x, t)\right\|_{L^{2}}^{2} d t \leq C .
\end{aligned}
$$

By (1.2) and Lemma 2.6 we obtain

$$
\begin{aligned}
\left\|v_{t x}\right\|_{L^{2}} \leq & C\left\|[\varphi(v)]_{x x}\right\|_{L^{2}}+\beta\left\|v_{x x x}\right\|_{L^{2}}+\left\|n_{x x}\right\|_{L^{2}}+\left\||\varepsilon|_{x x x}\right\|_{L^{2}} \\
\leq & C\left\|[\varphi(v)]_{x x}\right\|_{L^{2}}+\beta\left\|v_{x x x}\right\|_{L^{2}}+\left\|n_{x x}\right\|_{L^{2}} \\
& +\left\|\left(\varepsilon_{x x} \bar{\varepsilon}+2 \varepsilon \bar{\varepsilon}_{x}+\varepsilon \bar{\varepsilon}_{x x}\right)_{x}\right\|_{L^{2}} \\
\leq & C\left(\left\|v_{x x x}\right\|_{L^{2}}+1\right) \\
\leq & C .
\end{aligned}
$$


By (4.7) and (4.8) we obtain

$$
\begin{aligned}
& \sup _{t \in[0, T]}\left(\|\varepsilon\|_{H^{4}}+\|v\|_{H^{3}}+\|n\|_{H^{3}}+\left\|\varepsilon_{t}\right\|_{H^{2}}+\left\|v_{t}\right\|_{H^{1}}+\left\|n_{t}\right\|_{H^{2}}\right) \\
& \quad+\beta \int_{0}^{T}\left\|v_{x^{4}}(x, t)\right\|_{L^{2}}^{2} d t \leq C
\end{aligned}
$$

Corollary 4.1 Suppose that the conditions of Lemma 4.1 are satisfied. Then we have

$$
\sup _{t \in[0, T]}\left(\left\|E_{x x x}\right\|_{L^{\infty}}+\left\|v_{x x}\right\|_{L^{\infty}}+\left\|n_{x x}\right\|_{L^{\infty}}+\left\|\varepsilon_{t x}\right\|_{L^{\infty}}+\left\|n_{t x}\right\|_{L^{\infty}}+\left\|v_{t}\right\|_{L^{\infty}}\right) \leq C .
$$

Proof By Lemmas 2.3 and 4.1 the result of Corollary 4.1 is obvious.

Theorem 4.1 Suppose that the conditions of Lemma 4.1 are satisfied. Then there exists a unique global generalized solution of the initial boundary value problem (1.1)-(1.5), and

$$
\begin{aligned}
& \varepsilon(x, t) \in L^{\infty}\left(0, T ; H^{4}[0, L]\right), \quad E_{t}(x, t) \in L^{\infty}\left(0, T ; H^{2}[0, L]\right), \\
& v(x, t) \in L^{\infty}\left(0, T ; H^{3}[0, L]\right) \cap L^{2}\left(0, T ; H^{4}[0, L]\right), \quad v_{t}(x, t) \in L^{\infty}\left(0, T ; H^{1}[0, L]\right), \\
& n(x, t) \in L^{\infty}\left(0, T ; H^{3}[0, L]\right), \quad n_{t}(x, t) \in L^{\infty}\left(0, T ; H^{2}[0, L]\right) .
\end{aligned}
$$

Proof By using Theorem 3.1, Lemma 4.1, and Corollary 4.1 we can easily get this theorem.

Theorem 4.2 Suppose that the conditions of Lemma 4.1 are satisfied. Then there exists a unique global classical solution of the boundary value problem (1.1)-(1.5).

Proof By using Theorem 4.1 and the embedding theorems of Sobolev spaces we can easily get this theorem.

Theorem 4.3 Suppose that the conditions of Lemma 2.7 are satisfied. Then there exists a unique global smooth solution of the initial boundary value problem (1.1)-(1.5), and

$$
\begin{array}{ll}
\varepsilon(x, t) \in L^{\infty}\left(0, T ; H^{l+2}[0, L]\right), & \varepsilon_{t}(x, t) \in L^{\infty}\left(0, T ; H^{l}[0, L]\right), \\
v(x, t) \in L^{\infty}\left(0, T ; H^{l+1}[0, L]\right), & v_{t}(x, t) \in L^{\infty}\left(0, T ; H^{l-1}[0, L]\right) \cap L^{2}\left(0, T ; H^{l+2}[0, L]\right), \\
n(x, t) \in L^{\infty}\left(0, T ; H^{l+1}[0, L]\right), & n_{t}(x, t) \in L^{\infty}\left(0, T ; H^{l}[0, L]\right) .
\end{array}
$$

Proof By Lemma 2.7 and the embedding theorems of Sobolev spaces the result of Theorem 4.3 is obvious.

\section{Approximation of solution}

We now suppose that the generalized solution of initial boundary value problem (1.1)(1.5) is approximated by the generalized solution of the following problem:

$$
\begin{aligned}
& i \eta_{t}+\eta_{x x}+(\alpha-m) \eta=0, \\
& u_{t}+\left[\varphi(u)-\beta u_{x}+m+|\eta|^{2}\right]_{x}=0,
\end{aligned}
$$




$$
m_{t}+u_{x}=0, \quad t>0, x \in[0, L]
$$

with initial data

$$
\left.\eta\right|_{t=0}=\eta_{0}(x),\left.\quad u\right|_{t=0}=u_{0}(x),\left.\quad m\right|_{t=0}=m_{0}(x), \quad x \in[0, L],
$$

and boundary conditions

$$
\eta(0, t)=\eta(L, t)=u(0, t)=u(L, t)=m(0, t)=m(L, t)=0,
$$

where the parameters $p>0, \beta>0$, and $\alpha$ are real numbers, and $\varphi(s)$ is a real function.

Letting $F(t, x)=\varepsilon(t, x)-\eta(t, x), G(t, x)=v(t, x)-u(t, x), H(t, x)=n(t, x)-m(t, x)$, we obtain

$$
\begin{aligned}
& i F_{t}+F_{x x}+\alpha F-(H \varepsilon+m F)+\delta|\varepsilon|^{p} \varepsilon=0, \\
& G_{t}+[\varphi(v)-\varphi(u)]_{x}-\beta G_{x x}+H_{x}+\left(|\varepsilon|^{2}-|\eta|^{2}\right)_{x}=0, \\
& H_{t}+G_{x}=0, \quad t>0, x \in[0, L],
\end{aligned}
$$

with initial data

$$
\left.F\right|_{t=0}=0,\left.\quad G\right|_{t=0}=0,\left.\quad H\right|_{t=0}=0, \quad x \in[0, L],
$$

and boundary conditions

$$
F(0, t)=F(L, t)=G(0, t)=G(L, t)=H(0, t)=H(L, t)=0,
$$

where the parameters $p>0, \beta>0, \alpha$, and $\delta$ are real numbers, and $\varphi(s)$ is a real function.

Lemma 5.1 Suppose that the conditions of Theorem 3.1 are satisfied. Then for the solution of problem (5.6)-(5.10), we have

$$
\|F\|_{H^{2}}^{2}+\|G\|_{H^{1}}^{2}+\|H\|_{H^{1}}^{2}+\left\|F_{t}\right\|_{L^{2}}^{2}+\left\|H_{t}\right\|_{L^{2}}^{2} \leq \frac{C|\delta|}{|\delta|+1} e^{C(|\delta|+1) t}+C|\delta|^{2} .
$$

Proof Taking the inner product of (5.6) and $F$, it follows that

$$
\left(i F_{t}+F_{x x}+\alpha F-H \varepsilon-m F+\delta|\varepsilon|^{p} \varepsilon, F\right)=0 .
$$

Since

$$
\begin{aligned}
& \operatorname{Im}\left(i F_{t}, F\right)=\frac{1}{2} \frac{d}{d t}\|F\|_{L^{2}}^{2}, \quad \operatorname{Im}\left(F_{x x}+\alpha F, F\right)=0 \\
& |\operatorname{Im}(H \varepsilon+m F, F)| \leq C\|\varepsilon\|_{L^{\infty}}\|H\|_{L^{2}}\|F\|_{L^{2}} \leq C\left(\|H\|_{L^{2}}^{2}+\|F\|_{L^{2}}^{2}\right) \\
& \left|\operatorname{Im}\left(\delta|\varepsilon|^{p} \varepsilon, F\right)\right| \leq|\delta|\|\varepsilon\|_{L^{2 p+2}}^{p+1}\|F\|_{L^{2}} \\
& \leq|\delta|\left\|\varepsilon_{x}\right\|_{L^{2}}^{\frac{p}{2}}\|\varepsilon\|_{L^{2}}^{\frac{p+1}{2}}\|F\|_{L^{2}} \\
& \leq C|\delta|\left(\|F\|_{L^{2}}^{2}+1\right),
\end{aligned}
$$


from (5.11) we get

$$
\frac{d}{d t}\|F\|_{L^{2}}^{2} \leq C\left[(|\delta|+1)\left(\|F\|_{L^{2}}^{2}+\|H\|_{L^{2}}^{2}\right)+|\delta|\right]
$$

Taking the inner product of (5.7) and G, it follows that

$$
\left(G_{t}+[\varphi(v)-\varphi(u)]_{x}-\beta G_{x x}+H_{x}+\left(|\varepsilon|^{2}-|\eta|^{2}\right)_{x}, G\right)=0 .
$$

Since

$$
\begin{aligned}
& \left(G_{t}, G\right)=\frac{1}{2} \frac{d}{d t}\|G\|_{L^{2}}^{2}, \quad\left(-\beta G_{x x}, G\right)=\beta\left\|G_{x}\right\|_{L^{2}}^{2}, \\
& \left|\left([\varphi(v)]_{x}-[\varphi(u)]_{x}, G\right)\right|=\left|\left(\varphi^{\prime}(\xi)(v-u), G_{x}\right)\right| \\
& \leq\left\|\varphi^{\prime}(\xi)\right\|_{L^{\infty}}\|G\|_{L^{2}}\left\|G_{x}\right\|_{L^{2}} \\
& \leq C\left(|\xi|^{q}+1\right)\|G\|_{L^{2}}\left\|G_{x}\right\|_{L^{2}} \\
& \leq C\left(\|v\|_{L^{\infty}}^{q}+\|u\|_{L^{\infty}}^{q}+1\right)\|G\|_{L^{2}}\left\|G_{x}\right\|_{L^{2}} \\
& \leq C\left(\|G\|_{L^{2}}^{2}+\left\|H_{t}\right\|_{L^{2}}^{2}\right) \text {, } \\
& \left|\left(H_{x}, G\right)\right| \leq \frac{1}{2}\left(\|H\|_{L^{2}}^{2}+\left\|H_{t}\right\|_{L^{2}}^{2}\right), \\
& \left(|\varepsilon|_{x}^{2}-|\eta|_{x}^{2}, G\right)=\left|\int_{0}^{L}\left(|\varepsilon|^{2}-|\eta|^{2}\right) G_{x} d x\right| \\
& =\left|\int_{0}^{L}(F \bar{\varepsilon}+\eta \bar{F}) G_{x} d x\right| \\
& \leq\left(\|\varepsilon\|_{L^{\infty}}+\|\eta\|_{L^{\infty}}\right)\|F\|_{L^{2}}\left\|H_{t}\right\|_{L^{2}} \\
& \leq C\left(\|F\|_{L^{2}}^{2}+\left\|H_{t}\right\|_{L^{2}}^{2}\right) \text {, }
\end{aligned}
$$

from (5.13) we get

$$
\frac{d}{d t}\|G\|_{L^{2}}^{2} \leq C\left(\|G\|_{L^{2}}^{2}+\|F\|_{L^{2}}^{2}+\|H\|_{L^{2}}^{2}+\left\|H_{t}\right\|_{L^{2}}^{2}\right) .
$$

Since

$$
\frac{d}{d t}\|H\|_{L^{2}}^{2}=\frac{d}{d t} \int_{0}^{L} H^{2} d x \leq\|H\|_{L^{2}}^{2}+\left\|H_{t}\right\|_{L^{2}}^{2},
$$

by (5.12), (5.14), and (5.15) we get

$$
\begin{aligned}
\frac{d}{d t}\left(\|F\|_{L^{2}}^{2}+\|G\|_{L^{2}}^{2}+\|H\|_{L^{2}}^{2}\right) \leq & C(|\delta|+1)\left(\|F\|_{L^{2}}^{2}+\|G\|_{L^{2}}^{2}\right) \\
& +C(|\delta|+1)\left(\|H\|_{L^{2}}^{2}+\left\|H_{t}\right\|_{L^{2}}^{2}\right) \\
& +C|\delta| .
\end{aligned}
$$

Differentiating (5.6) with respect to $t$, we get

$$
i F_{t t}+F_{t x x}+\alpha F_{t}-(H \varepsilon+m F)_{t}+\delta\left(|\varepsilon|^{p} \varepsilon\right)_{t}=0 .
$$


Taking the inner product of (5.17) and $G_{t}$, it follows that

$$
\left(i F_{t t}+F_{t x x}+\alpha F_{t}-(H \varepsilon+m F)_{t}+\delta\left(|\varepsilon|^{p} \varepsilon\right)_{t}, F_{t}\right)=0
$$

Since

$$
\begin{aligned}
\operatorname{Im}\left(i F_{t t}, F_{t}\right)=\frac{1}{2} \frac{d}{d t}\left\|F_{t}\right\|_{L^{2}}^{2}, \quad \operatorname{Im}\left(F_{x x t}+\alpha F_{t}, F_{t}\right)=0 & \\
\left|\operatorname{Im}\left((H \varepsilon+m F)_{t}, F_{t}\right)\right|= & \left|\operatorname{Im} \int_{0}^{L}\left(H_{t} \varepsilon+H \varepsilon_{t}+m_{t} F+m F_{t}\right) \bar{F}_{t} d x\right| \\
= & \left|\operatorname{Im} \int_{0}^{L}\left(H_{t} \varepsilon+H \varepsilon_{t}+m_{t} F\right) \bar{F}_{t} d x\right| \\
\leq & \|\varepsilon\|_{L^{\infty}}\left\|H_{t}\right\|_{L^{2}}\left\|F_{t}\right\|_{L^{2}}+\left\|\varepsilon_{t}\right\|_{L^{\infty}}\|H\|_{L^{2}}\left\|F_{t}\right\|_{L^{2}} \\
& +\left\|m_{t}\right\|_{L^{\infty}}\|F\|_{L^{2}}\left\|F_{t}\right\|_{L^{2}} \\
\leq & C\left(\|H\|_{L^{2}}^{2}+\left\|H_{t}\right\|_{L^{2}}^{2}+\|F\|_{L^{2}}^{2}+\left\|F_{t}\right\|_{L^{2}}^{2}\right), \\
\left|\operatorname{Im}\left(\delta\left(|\varepsilon|^{p} \varepsilon\right)_{t}, F_{t}\right)\right| \leq & |\delta|\left|\operatorname{Im} \int_{0}^{L}\left(|\varepsilon|^{p} \varepsilon\right)_{t} \bar{F}_{t} d x\right| \\
= & \operatorname{Im} \int_{0}^{L}\left[\left(1+\frac{p}{2}\right) \delta|\varepsilon|^{p} \varepsilon_{t}+\frac{p \delta}{2}|\varepsilon|^{p-2} \varepsilon^{2} \bar{\varepsilon}_{t}\right] \bar{F}_{t} d x \\
\leq & C|\delta|\left(\left\|F_{t}\right\|_{L^{2}}^{2}+1\right),
\end{aligned}
$$

from (5.18) we get

$$
\frac{d}{d t}\left\|F_{t}\right\|_{L^{2}}^{2} \leq C\left[(|\delta|+1)\left(\|F\|_{L^{2}}^{2}+\left\|F_{t}\right\|_{L^{2}}^{2}+\|H\|_{L^{2}}^{2}+\left\|H_{t}\right\|_{L^{2}}^{2}\right)+|\delta|\right] .
$$

Taking the inner product of (5.7) and $-G_{x x}$, it follows that

$$
\left(G_{t}+[\varphi(v)]_{x}-[\varphi(u)]_{x}-\beta G_{x x}+H_{x}+|\varepsilon|_{x}^{2}-|\eta|_{x}^{2},-G_{x x}\right)=0 .
$$

Since

$$
\begin{aligned}
\left(G_{t},-G_{x x}\right)=\frac{1}{2} \frac{d}{d t}\left\|G_{x}\right\|_{L^{2}}^{2}, & \quad\left(-\beta G_{x x},-G_{x x}\right)=\beta\left\|G_{x x}\right\|_{L^{2}}^{2}, \\
\left|\left([\varphi(v)]_{x}-[\varphi(u)]_{x},-G_{x x}\right)\right| & =\left|\int_{0}^{L}[\varphi(v)-\varphi(u)] G_{x x x} d x\right| \\
& =\left|\left(\varphi^{\prime}(\xi) G, G_{x x x}\right)\right| \\
& =\left\|\varphi^{\prime}(\xi)\right\|_{L^{\infty}}\left|\left(G_{x}, G_{x x}\right)\right| \\
& \leq\left\|\varphi^{\prime}(\xi)\right\|_{L^{\infty}}\left\|G_{x}\right\|_{L^{2}}\left\|G_{x x}\right\|_{L^{2}} \\
& \leq C\left(|\xi|^{q}+1\right)\left\|G_{x}\right\|_{L^{2}}\left\|G_{x x}\right\|_{L^{2}} \\
\leq & \leq\left(\|v\|_{L^{\infty}}^{q}+\|u\|_{L^{\infty}}^{q}+1\right)\left\|G_{x}\right\|_{L^{2}}\left\|G_{x x}\right\|_{L^{2}} \\
\leq & \frac{\beta}{2}\left\|G_{x x}\right\|_{L^{2}}^{2}+C\left\|G_{x}\right\|_{L^{2}}^{2},
\end{aligned}
$$




$$
\begin{aligned}
&\left(H_{x},-G_{x x}\right)=-\int_{0}^{L} H_{x} G_{x x} d x=\int_{0}^{L} H_{x} H_{x t} d x=\frac{1}{2} \frac{d}{d t}\left\|H_{x}\right\|_{L^{2}}^{2}, \\
&\left|\left(|\varepsilon|_{x}^{2}-|\eta|_{x}^{2},-G_{x x}\right)\right|=\left|\int_{0}^{L}\left(F_{x} \bar{\varepsilon}+\eta_{x} \bar{F}+F \bar{\varepsilon}_{x}+\eta \bar{F}_{x}\right) G_{x x} d x\right| \\
&=\left|\int_{0}^{L}\left(F_{x} \bar{\varepsilon}+\eta \bar{F}_{x}\right) G_{x x} d x\right| \\
&+\left|\int_{0}^{L}\left(F \bar{\varepsilon}_{x}+\eta_{x} \bar{F}_{x}\right) G_{x x} d x\right| \\
&=\left|\int_{0}^{L}\left(F_{x} \bar{\varepsilon}+\eta \bar{F}_{x}\right)_{x} G_{x} d x\right| \\
&+\left|\int_{0}^{L}\left(F \bar{\varepsilon}_{x}+\eta_{x} \bar{F}_{x}\right)_{x} G_{x} d x\right| \\
& \leq \\
& \quad \int_{0}^{L}(|\varepsilon|+|\eta|)\left|F_{x} \| G_{x x}\right| d x \\
&+\int_{0}^{L}\left(\left|\varepsilon_{x}\right|+\left|\eta_{x}\right|\right)\left|\varepsilon \| G_{x x}\right| d x \\
& \leq\left(\|\varepsilon\|_{L^{\infty}}+\|\eta\|_{L^{\infty}}\right)\left\|F_{x}\right\|_{L^{2}}\left\|G_{x x}\right\|_{L^{2}} \\
&+\left(\left\|\varepsilon_{x}\right\|_{L^{\infty}}+\left\|\eta_{x}\right\|_{L^{\infty}}\right)\|F\|_{L^{2}}\left\|G_{x x}\right\|_{L^{2}} \\
& \leq \frac{\beta}{2}\left\|G_{x x}\right\|_{L^{2}}^{2}+C\left(\|F\|_{L^{2}}^{2}+\left\|F_{x}\right\|_{L^{2}}^{2}\right),
\end{aligned}
$$

from (5.8) and (5.20) we get

$$
\begin{aligned}
\frac{d}{d t}\left(\left\|H_{t}\right\|_{L^{2}}^{2}+\left\|H_{x}\right\|_{L^{2}}^{2}\right) & =\frac{d}{d t}\left(\left\|G_{x}\right\|_{L^{2}}^{2}+\left\|H_{x}\right\|_{L^{2}}^{2}\right) \\
& \leq C\left(\|F\|_{L^{2}}^{2}+\left\|F_{x}\right\|_{L^{2}}^{2}+\left\|G_{x}\right\|_{L^{2}}^{2}\right) .
\end{aligned}
$$

Taking the inner product of (5.11) and $F_{x x}$, it follows that

$$
\left(i F_{t}+F_{x x}+\alpha F-H \varepsilon-m F+\delta|\varepsilon|^{p} \varepsilon, F_{x x}\right)=0 .
$$

Since

$$
\begin{aligned}
& \operatorname{Im}\left(i F_{t}, F_{x x}\right)=\frac{1}{2} \frac{d}{d t}\left\|F_{x}\right\|_{L^{2}}^{2}, \quad \operatorname{Im}\left(F_{x x}+\alpha F, F_{x x}\right)=0, \\
& \left|\operatorname{Im}\left(H \varepsilon+m F, F_{x x}\right)\right|=\left|\operatorname{Im} \int_{0}^{L}(H \varepsilon+m F)_{x} F_{x} d x\right| \\
& \leq\left|\operatorname{Im} \int_{0}^{L}\left(H_{x} \varepsilon+H \varepsilon_{x}+m_{x} F+m F_{x}\right) F_{x} d x\right| \\
& \leq C\left(\|\varepsilon\|_{L^{\infty}}\left\|H_{x}\right\|_{L^{2}}+\left\|\varepsilon_{x}\right\|_{L^{\infty}}\|H\|_{L^{2}}\right)\left\|F_{x}\right\|_{L^{2}} \\
& \\
& +C\left(\left\|m_{x}\right\|_{L^{\infty}}\|F\|_{L^{2}}+\|m\|_{L^{\infty}}\left\|F_{x}\right\|_{L^{2}}\right)\left\|F_{x}\right\|_{L^{2}} \\
& \leq C\left(\|H\|_{L^{2}}^{2}+\left\|H_{x}\right\|_{L^{2}}^{2}+\|F\|_{L^{2}}^{2}+\left\|F_{x}\right\|_{L^{2}}^{2}\right),
\end{aligned}
$$




$$
\begin{aligned}
\left|\operatorname{Im}\left(\delta|\varepsilon|^{p} \varepsilon, F_{x x}\right)\right| & =\left|\operatorname{Im} \int_{0}^{L} \delta\left(|\varepsilon|^{p} \varepsilon\right)_{x} F_{x} d x\right| \\
& \leq|\delta| \int_{0}^{L}\left|\left(|\varepsilon|_{x}^{p} \varepsilon+|\varepsilon|^{p} \varepsilon_{x}\right)\right|\left|F_{x}\right| d x \\
& \leq|\delta| \int_{0}^{L}\left|\left[\frac{p}{2}|\varepsilon|^{p-2}\left(\varepsilon_{x} \bar{\varepsilon}+\varepsilon \bar{\varepsilon}_{x}\right)+|\varepsilon|^{p} \varepsilon_{x}\right]\right|\left|F_{x}\right| d x \\
& \leq C|\delta|\left(\left\|F_{x}\right\|_{L^{2}}^{2}+1\right),
\end{aligned}
$$

from (5.22), we get

$$
\frac{d}{d t}\left\|F_{x}\right\|_{L^{2}}^{2} \leq C\left[(|\delta|+1)\left(\|H\|_{L^{2}}^{2}+\left\|H_{x}\right\|_{L^{2}}^{2}+\|F\|_{L^{2}}^{2}+\left\|F_{x}\right\|_{L^{2}}^{2}\right)+|\delta|\right] .
$$

By (5.16), (5.19), (5.21), and (5.23) we obtain

$$
\begin{aligned}
& \frac{d}{d t}\left(\|F\|_{L^{2}}^{2}+\left\|F_{x}\right\|_{L^{2}}^{2}+\left\|F_{t}\right\|_{L^{2}}^{2}+\|G\|_{L^{2}}^{2}+\|H\|_{L^{2}}^{2}+\left\|H_{t}\right\|_{L^{2}}^{2}+\left\|H_{x}\right\|_{L^{2}}^{2}\right) \\
& \leq C(|\delta|+1)\left(\|F\|_{L^{2}}^{2}+\left\|F_{x}\right\|_{L^{2}}^{2}+\left\|F_{t}\right\|_{L^{2}}^{2}\right)+C|\delta| \\
& \quad+C(|\delta|+1)\left(\|G\|_{L^{2}}^{2}+\|H\|_{L^{2}}^{2}+\left\|H_{t}\right\|_{L^{2}}^{2}+\left\|H_{x}\right\|_{L^{2}}^{2}\right)
\end{aligned}
$$

By using Gronwall's inequality we obtain

$$
\begin{aligned}
& \|F\|_{L^{2}}^{2}+\left\|F_{x}\right\|_{L^{2}}^{2}+\left\|F_{t}\right\|_{L^{2}}^{2}+\|G\|_{L^{2}}^{2}+\|H\|_{L^{2}}^{2}+\left\|H_{x}\right\|_{L^{2}}^{2}+\left\|H_{t}\right\|_{L^{2}}^{2} \\
& \quad \leq \frac{C|\delta|}{|\delta|+1} e^{C(|\delta|+1) t} .
\end{aligned}
$$

By (5.8) we get

$$
\begin{gathered}
\|F\|_{L^{2}}^{2}+\left\|F_{x}\right\|_{L^{2}}^{2}+\left\|F_{t}\right\|_{L^{2}}^{2}+\|G\|_{L^{2}}^{2}+\left\|G_{x}\right\|_{L^{2}}^{2}+\|H\|_{L^{2}}^{2} \\
+\left\|H_{t}\right\|_{L^{2}}^{2}+\left\|H_{x}\right\|_{L^{2}}^{2} \leq \frac{C|\delta|}{|\delta|+1} e^{C(|\delta|+1) t}
\end{gathered}
$$

By (5.6) and (5.24) we obtain

$$
\begin{aligned}
\left\|F_{x x}\right\|_{L^{2}}^{2} \leq & {\left[|\alpha|\|F\|_{L^{2}}+\|H \varepsilon+m F\|_{L^{2}}+\left\|F_{t}\right\|_{L^{2}}+\left\|\delta|\varepsilon|^{p} \varepsilon\right\|_{L^{2}}\right]^{2} } \\
\leq & C\left[|\alpha|^{2}\|F\|_{L^{2}}^{2}+\|H \varepsilon\|_{L^{2}}^{2}+\|m F\|_{L^{2}}^{2}\right. \\
& \left.+\left\|F_{t}\right\|_{L^{2}}^{2}+|\delta|^{2}\left(\|\varepsilon\|_{L^{2 p+2}}^{2 p+2}\right)^{2}\right] \\
\leq & C\left[|\alpha|^{2}\|F\|_{L^{2}}^{2}+\|\varepsilon\|_{L^{\infty}}\|H\|_{L^{2}}^{2}+\|m\|_{L^{\infty}}\|F\|_{L^{2}}^{2}\right. \\
& \left.+\left\|F_{t}\right\|_{L^{2}}^{2}+|\delta|^{2}\left(C\left\|\varepsilon_{x}\right\|_{L^{2}}^{\frac{p}{2}}\|\varepsilon\|_{L^{2}}^{\frac{p+4}{2(p+2)}}\right)^{2}\right] \\
\leq & C\left[|\alpha|^{2}\|F\|_{L^{2}}^{2}+\|\varepsilon\|_{L^{\infty}}\|H\|_{L^{2}}^{2}+\|m\|_{L^{\infty}}\|F\|_{L^{2}}^{2}\right. \\
& \left.+\left\|F_{t}\right\|_{L^{2}}^{2}+C|\delta|^{2}\right] \\
\leq & C\left(\|F\|_{L^{2}}^{2}+\left\|F_{t}\right\|_{L^{2}}^{2}+\|H\|_{L^{2}}^{2}+|\delta|^{2}\right) \\
\leq & \frac{C|\delta|}{|\delta|+1} e^{C(|\delta|+1) t}+C|\delta|^{2} .
\end{aligned}
$$


By (5.24) and (5.25) we obtain

$$
\|F\|_{H^{2}}^{2}+\|G\|_{H^{1}}^{2}+\|H\|_{H^{1}}^{2}+\left\|F_{t}\right\|_{L^{2}}^{2}+\left\|H_{t}\right\|_{L^{2}}^{2} \leq \frac{C|\delta|}{|\delta|+1} e^{C(|\delta|+1) t}+C|\delta|^{2} .
$$

Lemma 5.2 Suppose that the conditions of Theorem 3.1 are satisfied. Then for the solution of problem (5.6)-(5.10), we have

$$
\begin{aligned}
& \|F\|_{H^{3}}^{2}+\|G\|_{H^{2}}^{2}+\|H\|_{H^{2}}^{2}+\left\|F_{t}\right\|_{H^{1}}^{2}+\left\|G_{t}\right\|_{L^{2}}^{2}+\left\|H_{t}\right\|_{H^{1}}^{2} \\
& \quad \leq \frac{C|\delta|}{|\delta|+1} e^{C(|\delta|+1)}+C|\delta|^{2} e^{C t}+C|\delta|^{2} .
\end{aligned}
$$

Proof Taking the inner product of (5.17) and $-F_{t x x}$, it follows that

$$
\left(i F_{t t}+F_{t x x}+\alpha F_{t}-(H \varepsilon+m F)_{t}+\delta\left(|\varepsilon|^{p} \varepsilon\right)_{t},-F_{t x x}\right)=0
$$

Since

$$
\begin{aligned}
& \operatorname{Im}\left(i F_{t t},-F_{t x x}\right)=\frac{1}{2} \frac{d}{d t}\left\|F_{t x}\right\|_{L^{2}}^{2}, \quad \operatorname{Im}\left(F_{x x t}+\alpha F_{t},-F_{t x x}\right)=0, \\
& \left|\operatorname{Im}\left((H \varepsilon+m F)_{t},-F_{t x x}\right)\right|=\left|\operatorname{Im} \int_{0}^{L}\left(H_{t} \varepsilon+H \varepsilon_{t}+m_{t} F+m F_{t}\right)_{x} \bar{F}_{t x} d x\right| \\
& =\left|\operatorname{Im} \int_{0}^{L}\left(H_{x t} \varepsilon+H_{t} \varepsilon_{x}\right) \bar{F}_{t x} d x\right|+\left|\operatorname{Im} \int_{0}^{L}\left(H_{x} \varepsilon_{t}+H \varepsilon_{t x}\right) \bar{F}_{t x} d x\right| \\
& +\left|\operatorname{Im} \int_{0}^{L}\left(m_{x t} F+m_{t} F_{x}\right) \bar{F}_{t x} d x\right| \\
& +\left|\operatorname{Im} \int_{0}^{L}\left(m_{x} F_{t}+m F_{x t}\right) \bar{F}_{t x} d x\right| \\
& \leq C\left(\|F\|_{L^{2}}^{2}+\left\|F_{x}\right\|_{L^{2}}^{2}+\left\|F_{t}\right\|_{L^{2}}^{2}\right) \\
& +C\left(\|H\|_{L^{2}}^{2}+\left\|H_{x}\right\|_{L^{2}}^{2}+\left\|H_{t}\right\|_{L^{2}}^{2}\right)+C\left(\left\|F_{t x}\right\|_{L^{2}}^{2}+\left\|H_{t x}\right\|_{L^{2}}^{2}\right) \\
& \leq \frac{C|\delta|}{|\delta|+1} e^{C(|\delta|+1) t}+C|\delta|^{2}+C\left(\left\|F_{t x}\right\|_{L^{2}}^{2}+\left\|H_{t x}\right\|_{L^{2}}^{2}\right), \\
& \operatorname{Im}\left(\left(\delta|\varepsilon|^{p} \varepsilon\right)_{t},-F_{t x x}\right)=-\operatorname{Im} \int_{0}^{L}\left[\left(1+\frac{p}{2}\right) \delta|\varepsilon|^{p} \varepsilon_{t}+\frac{p \delta}{2}|\varepsilon|^{p-2} \varepsilon^{2} \bar{\varepsilon}_{t}\right] \bar{F}_{t x x} d x \\
& =\operatorname{Im} \int_{0}^{L}\left[\left(1+\frac{p}{2}\right) \delta|\varepsilon|^{p} \varepsilon_{t}+\frac{p \delta}{2}|\varepsilon|^{p-2} \varepsilon^{2} \bar{\varepsilon}_{t}\right]_{x} \bar{F}_{t x} d x \\
& =\operatorname{Im} \int_{0}^{L}\left[\left(1+\frac{p}{2}\right) \delta|\varepsilon|_{x}^{p} \varepsilon_{t}+\left(1+\frac{p}{2}\right) \delta|\varepsilon|^{p} \varepsilon_{t x}\right] \bar{F}_{t x} d x \\
& +\operatorname{Im} \int_{0}^{L}\left(\frac{p \delta}{2}|\varepsilon|_{x}^{p-2} \varepsilon^{2} \bar{\varepsilon}_{t}+\frac{p \delta}{2}|\varepsilon|^{p-2} \varepsilon_{x}^{2} \bar{\varepsilon}_{t} \bar{\varepsilon}_{t x}\right) \bar{F}_{t x} d x \\
& +\operatorname{Im} \int_{0}^{L} \frac{p \delta}{2}|\varepsilon|^{p-2} \varepsilon^{2} \bar{\varepsilon}_{t x} \bar{F}_{t x} d x \\
& \leq C|\delta|\left(\left\|F_{t x}\right\|_{L^{2}}^{2}+1\right) \text {, }
\end{aligned}
$$


from (5.27) we get

$$
\frac{d}{d t}\left\|F_{t x}\right\|_{L^{2}}^{2} \leq C(|\delta|+1)\left(\left\|F_{t x}\right\|_{L^{2}}^{2}+\left\|H_{t x}\right\|_{L^{2}}^{2}\right)+\frac{C|\delta|}{|\delta|+1} e^{C(|\delta|+1) t}+C|\delta|^{2}
$$

Taking the inner product of (5.7) and $G_{x^{4}}$, it follows that

$$
\left(G_{t}+[\varphi(v)-\varphi(u)]_{x}-\beta G_{x x}+H_{x}+\left(|\varepsilon|^{2}-|\eta|^{2}\right)_{x}, G_{x^{4}}\right)=0 .
$$

Since

$$
\begin{aligned}
& \left(G_{t}, G_{x^{4}}\right)=\frac{1}{2} \frac{d}{d t}\left\|G_{x x}\right\|_{L^{2}}^{2}, \quad\left(-\beta G_{x x}, G_{x^{4}}\right)=\beta\left\|G_{x x x}\right\|_{L^{2}}^{2}, \\
& \left|\left([\varphi(v)-\varphi(u)]_{x}, G_{x^{4}}\right)\right|=\left|\int_{0}^{L}[\varphi(v)-\varphi(u)]_{x} G_{x^{4}} d x\right| \\
& =\left|\int_{0}^{L}[\varphi(v)-\varphi(u)] G_{x^{5}} d x\right| \\
& =\left|\int_{0}^{L} \varphi^{\prime}(\xi) G G_{x^{5}} d x\right| \\
& \leq\left\|\varphi^{\prime}(\xi)\right\|_{L^{\infty}}\|G\|_{L^{2}}\left\|G_{x^{5}}\right\|_{L^{2}} \\
& \leq\left\|\varphi^{\prime}(\xi)\right\|_{L^{\infty}}\left\|G_{x x}\right\|_{L^{2}}\left\|G_{x x x}\right\|_{L^{2}} \\
& \leq C\left(|\xi|^{q}+1\right)\left\|G_{x x}\right\|_{L^{2}}\left\|G_{x x x}\right\|_{L^{2}} \\
& \leq C\left(\|v\|_{L^{\infty}}^{q}+\|u\|_{L^{\infty}}^{q}+1\right)\left\|G_{x x}\right\|_{L^{2}}\left\|G_{x x x}\right\|_{L^{2}} \\
& \leq \frac{\beta}{4}\left\|G_{x x x}\right\|_{L^{2}}^{2}+C\left\|G_{x x}\right\|_{L^{2}}^{2} \text {, } \\
& \left(H_{x}, G_{x^{4}}\right)=-\int_{0}^{2 L} H_{x x} G_{x x x} d x=\int_{0}^{2 L} H_{x x} H_{x x t} d x=\frac{1}{2} \frac{d}{d t}\left\|H_{x x}\right\|_{L^{2}}^{2} \text {, } \\
& \left|\left(\left(|\varepsilon|^{2}-|\eta|^{2}\right)_{x}, G_{x^{4}}\right)\right|=\left|\int_{0}^{L}\left(|\varepsilon|^{2}-|\eta|^{2}\right)_{x} G_{x^{4}} d x\right| \\
& =\left|\int_{0}^{L}\left(F_{x} \bar{\varepsilon}+\eta_{x} \bar{F}+F \bar{\varepsilon}_{x}+\eta \bar{F}_{x}\right) G_{x x x} d x\right| \\
& \leq \frac{\beta}{4}\left\|G_{x x x}\right\|_{L^{2}}^{2}+C\left(\|F\|_{L^{2}}^{2}+\left\|F_{x}\right\|_{L^{2}}^{2}\right) \\
& \leq \frac{\beta}{4}\left\|G_{x x x}\right\|_{L^{2}}^{2}+\frac{C|\delta|}{|\delta|+1} e^{C(|\delta|+1) t}+C|\delta|^{2},
\end{aligned}
$$

from (5.29) we get

$$
\begin{aligned}
\frac{d}{d t}\left(\left\|G_{x x}\right\|_{L^{2}}^{2}+\left\|H_{x x}\right\|_{L^{2}}^{2}\right) \leq & C\left(\left\|G_{x x}\right\|_{L^{2}}^{2}+\left\|H_{x x}\right\|_{L^{2}}^{2}\right) \\
& +\frac{C|\delta|}{|\delta|+1} e^{C(|\delta|+1) t}+C|\delta|^{2}
\end{aligned}
$$


By (5.28) and (5.30) we obtain

$$
\begin{aligned}
\frac{d}{d t}\left(\left\|F_{t x}\right\|_{L^{2}}^{2}+\left\|G_{x x}\right\|_{L^{2}}^{2}+\left\|H_{x x}\right\|_{L^{2}}^{2}\right) \leq & C\left(\left\|F_{t x}\right\|_{L^{2}}^{2}+\left\|G_{x x}\right\|_{L^{2}}^{2}+\left\|H_{x x}\right\|_{L^{2}}^{2}\right) \\
& +\frac{C|\delta|}{|\delta|+1} e^{C(|\delta|+1) t}+C|\delta|^{2}
\end{aligned}
$$

and thus by Gronwall's inequality we obtain

$$
\begin{aligned}
\left\|F_{t x}\right\|_{L^{2}}^{2}+\left\|G_{x x}\right\|_{L^{2}}^{2}+\left\|H_{x x}\right\|_{L^{2}}^{2} & \leq \frac{C|\delta|}{(|\delta|+1)^{2}} e^{C(|\delta|+1) t} e^{C t}+C|\delta|^{2} e^{C t} \\
& \leq \frac{C|\delta|}{|\delta|+1} e^{C(|\delta|+1) t}+C|\delta|^{2} e^{C t} .
\end{aligned}
$$

By (5.8) we obtain

$$
\left\|F_{t x}\right\|_{L^{2}}^{2}+\left\|G_{x x}\right\|_{L^{2}}^{2}+\left\|H_{x x}\right\|_{L^{2}}^{2}+\left\|H_{t x}\right\|_{L^{2}}^{2} \leq \frac{C|\delta|}{|\delta|+1} e^{C(|\delta|+1) t}+C|\delta|^{2} e^{C t} .
$$

By (5.6), Young's inequality, and (5.32) we obtain

$$
\begin{aligned}
\left\|F_{x x x}\right\|_{L^{2}}^{2} \leq & C\left[|\alpha|\left\|F_{x}\right\|_{L^{2}}^{2}+\left\|(H \varepsilon+m F)_{x}\right\|_{L^{2}}^{2}\right] \\
& +C\left[\left\|F_{t x}\right\|_{L^{2}}^{2}+\left\|\delta\left(|\varepsilon|^{p} \varepsilon\right)_{x}\right\|_{L^{2}}^{2}\right] \\
\leq & C\left\|F_{x}\right\|_{L^{2}}^{2}+C\left\|F_{t x}\right\|_{L^{2}}^{2} \\
& +C\left(\left\|H_{x} \varepsilon\right\|_{L^{2}}+\left\|H \varepsilon_{x}\right\|_{L^{2}}+\left\|m_{x} F\right\|_{L^{2}}+\left\|m F_{x}\right\|_{L^{2}}\right)^{2} \\
& +C\left\|\left(1+\frac{p}{2}\right) \delta|\varepsilon|^{p} \varepsilon_{x}+\frac{p \delta}{2}|\varepsilon|^{p-2} \varepsilon^{2} \bar{\varepsilon}_{x}\right\|_{L^{2}}^{2} \\
\leq & C\left\|F_{x}\right\|_{L^{2}}^{2}+C\left\|F_{t x}\right\|_{L^{2}}^{2} \\
& +C\left(\left\|H_{x} \varepsilon\right\|_{L^{2}}^{2}+\left\|H \varepsilon_{x}\right\|_{L^{2}}^{2}+\left\|m_{x} F\right\|_{L^{2}}^{2}+\left\|m F_{x}\right\|_{L^{2}}^{2}\right) \\
& +C\left(1+\frac{p^{2}}{4}\right)|\delta|^{2}\left\||\varepsilon|^{p} \varepsilon_{x}\right\|_{L^{2}}^{2}+C \frac{p^{2}|\delta|^{2}}{4}\left\||\varepsilon|^{p} \varepsilon_{x}\right\|_{L^{2}}^{2} \\
\leq & C\left(\|F\|_{L^{2}}^{2}+\left\|F_{x}\right\|_{L^{2}}^{2}+\left\|F_{t x}\right\|_{L^{2}}^{2}\right) \\
& +C\left(\|H\|_{L^{2}}^{2}+\left\|H_{x}\right\|_{L^{2}}^{2} \|_{L^{2}}\right)+C|\delta|^{2} \\
\leq & \frac{C|\delta|}{|\delta|+1} e^{C(|\delta|+1) t}+C|\delta|^{2} e^{C t}+C|\delta|^{2} .
\end{aligned}
$$

By (1.3), (5.32), and (5.33) we obtain

$$
\begin{gathered}
\left\|F_{x x x}\right\|_{L^{2}}^{2}+\left\|F_{t x}\right\|_{L^{2}}^{2}+\left\|G_{x x}\right\|_{L^{2}}^{2}+\left\|H_{x x}\right\|_{L^{2}}^{2}+\left\|H_{t x}\right\|_{L^{2}}^{2} \\
\leq \frac{C|\delta|}{|\delta|+1} e^{C(|\delta|+1) t}+C|\delta|^{2} e^{C t}+C|\delta|^{2}
\end{gathered}
$$


By (5.7) we obtain

$$
\begin{aligned}
\left\|G_{t}\right\|_{L^{2}}^{2} \leq & C\left[\left\|[\varphi(v)-\varphi(u)]_{x}\right\|_{L^{2}}^{2}+\beta\left\|G_{x x}\right\|_{L^{2}}^{2}+\left\|H_{x}\right\|_{L^{2}}^{2}\right. \\
& \left.+\left\|\left(|\varepsilon|^{2}-|\eta|^{2}\right)_{x}\right\|_{L^{2}}^{2}\right] \\
\leq & C\left[\left\|\varphi^{\prime}(\xi)\right\|_{L^{\infty}}\left\|G_{x}\right\|_{L^{2}}^{2}+\beta\left\|G_{x x}\right\|_{L^{2}}^{2}+\left\|H_{x}\right\|_{L^{2}}^{2}\right. \\
& \left.+\left\|\left(F_{x} \bar{\varepsilon}+\eta_{x} \bar{F}+F \bar{\varepsilon}_{x}+\eta \bar{F}_{x}\right)\right\|_{L^{2}}^{2}\right] \\
\leq & \frac{C|\delta|}{|\delta|+1} e^{C(|\delta|+1) t}+C|\delta|^{2} e^{C t}+C|\delta|^{2} .
\end{aligned}
$$

By (5.34) and (5.35) we obtain

$$
\begin{gathered}
\|F\|_{H^{3}}^{2}+\|G\|_{H^{2}}^{2}+\|H\|_{H^{2}}^{2}+\left\|F_{t}\right\|_{H^{1}}^{2}+\left\|G_{t}\right\|_{L^{2}}^{2}+\left\|H_{t}\right\|_{H^{1}}^{2} \\
\leq \frac{C|\delta|}{|\delta|+1} e^{C(|\delta|+1) t}+C|\delta|^{2} e^{C t}+C|\delta|^{2}
\end{gathered}
$$

By Lemmas 5.1 and 5.2 we obtain the following:

Theorem 5.1 Suppose that the conditions of Theorem 3.1 are satisfied. If $(\varepsilon, v, n)$ is the solution to problem (1.1)-(1.5) and $(\eta, u, m)$ is the solution to problem (5.1)-(5.5), then

$$
\|\varepsilon-\eta\|_{H^{3}}^{2}+\|v-u\|_{H^{2}}^{2}+\|n-m\|_{H^{2}}^{2} \leq \frac{C|\delta|}{|\delta|+1} e^{C(|\delta|+1) t}+C|\delta|^{2} e^{C t}+C|\delta|^{2},
$$

where $C$ is a positive constant.

\section{Acknowledgements}

The authors are grateful to the referees for useful remarks and suggestions that greatly improved the presentation of this manuscript.

\section{Funding}

This work is supported by the National Natural Science Foundation of China (Grant 11401223, 11871172), the National Natural Science Foundation of Guangdong (Grant 2015A030313424), and the Science and Technology Program of Guangzhou (Grant 201607010005).

\section{Abbreviations}

Not applicable.

\section{Availability of data and materials}

Not applicable.

\section{Competing interests}

The authors declare that they have no competing interests.

Authors' contributions

XW carried out the studies and drafted the manuscript. All authors read and approved the final version of the manuscript.

Author details

'College of Mathematics and Informatics, South China Agricultural University, Guangzhou, China. ${ }^{2}$ School of Mathematics and Information Science, Guangzhou University, Guangzhou, China.

\section{Publisher's Note}

Springer Nature remains neutral with regard to jurisdictional claims in published maps and institutional affiliations.

Received: 7 October 2019 Accepted: 23 April 2020 Published online: 06 May 2020 


\section{References}

1. Zakharov, V.E.: Collapse of Langmuir waves. Sov. Phys. JETP 35, 908-914 (1972)

2. Davydov, A.S.: Solitons in molecular systems. Phys. Scr. 20, 387-394 (1979)

3. Stenflo, L.: Nonlinear equations for acoustic gravity waves. Phys. Scr. 33, 156-158 (1986)

4. Ma, S., Chang, Q:: Stranger attractors on pseudospectral solutions for dissipative Zakharov equations. Acta Math. Sci. 24B, 321-336 (2004)

5. Pecher, H.: An improved local well-posedness result for the one-dimensional Zakharov system. J. Math. Anal. Appl. $342,1440-1454$ (2008)

6. Guo, B.L., Zhang, J.J., Pu, X.K.: On the existence and uniqueness of smooth solution for a generalized Zakharov equation. J. Math. Anal. Appl. 365, 238-253 (2010)

7. Linares, F., Matheus, C.: Well-posedness for the 1D Zakharov-Rubenchik system. Adv. Differ. Equ. 14, 261-288 (2009)

8. Linares, F., Saut, J.C.: The Cauchy problem for the 3D Zakharov-Kuznetsov equation. Discrete Contin. Dyn. Syst., Ser. A 24, 547-565 (2009)

9. Guo, B.L.: On some problems for a wide class of systems of Zakharov equations. In: Proceedings of DD-3, Changchun Symposium, pp. 395-415 (1982)

10. Guo, B.L., Shen, L.J.: The existence and uniqueness of the classical solution on the periodic initial problem for Zakharov equation. Acta Math. Appl. Sin. 5, 310-324 (1982)

11. Guo, B.L., Gan, Z.H., Kong, L.H., Zhang, J.J.: The Zakharov System and Its Soliton Solutions. Springer, Singapore (2016)

12. Zheng, X.X., Shang, Y.D., Di, H.F.: The time-periodic solutions to the modified Zakharov equations with a quantum correction. Mediterr. J. Math. 14, Article ID 152 (2017)

13. Wang, X.Q., Shang, Y.D.: On the global existence and small dissipation limit for generalized dissipative Zakharov system. Math. Methods Appl. Sci. 41, 3718-3749 (2018)

14. Gajewski, H., Zacharias, K.: Über Näherungsverfahren zur Lösung der nichtlinearen Schrödinger-Gleichung mit selbstkonsistentem Potential. Math. Nachr. 89,71-85 (1979)

15. You, S.J., Ning, X.Q.: On global smooth solution for generalized Zakharov equations. Comput. Math. Appl. 72, 64-75 (2016)

\section{Submit your manuscript to a SpringerOpen ${ }^{\circ}$ journal and benefit from:}

- Convenient online submission

- Rigorous peer review

- Open access: articles freely available online

- High visibility within the field

- Retaining the copyright to your article

Submit your next manuscript at $\gg$ springeropen.com 